\title{
A transmurally heterogeneous orthotropic activation model for ventricular contraction and its numerical validation
}

\author{
Luca Barbarotta $^{1}$ (I) $\mid$ Simone Rossi ${ }^{2}$ | Luca Dedè $^{3} \mid$ Alfio Quarteroni ${ }^{3,4}$
}

\author{
${ }^{1}$ Department of Biomedical Engineering, \\ Technische Universiteit Eindhoven, \\ Eindhoven, The Netherlands \\ ${ }^{2}$ Department of Mathematics, University \\ of North Carolina, Chapel Hill, North \\ Carolina \\ ${ }^{3}$ Dipartimento di Matematica, Politecnico \\ di Milano, Milan, Italy \\ ${ }^{4}$ Institute of Mathematics, Ećole \\ Polytechnique Fédérale de Lausanne, \\ Lausanne, Switzerland

\section{Correspondence} \\ Luca Barbarotta, Department of \\ Biomedical Engineering, Technische \\ Universiteit Eindhoven, PO Box 513, 5600 \\ MB Eindhoven, The Netherlands. \\ Email: 1.barbarotta@tue.nl
}

\begin{abstract}
Models for cardiac mechanics require an activation mechanism properly representing the stress-strain relations in the contracting myocardium. In this paper, we propose a new activation model that accounts for the transmural heterogeneities observed in myocardial strain measurements. In order to take the anisotropy of the active mechanics into account, our model is based on an active strain formulation. Thanks to multiplicative decomposition of the deformation gradient tensor, in this formulation, the active strains orthogonal to the fibers can be naturally described. We compare the results of our novel formulation against different anisotropic models of the active contraction of the cardiac muscle, as well as against experimental data available in the literature. We show that with the currently available models, the strain distributions are not in agreement with the reported experimental measurements. Conversely, we show that our new transmurally heterogeneous orthotropic activation model improves the accuracy of shear strains related to in-plane rotations and torsion.
\end{abstract}

\section{KEYWORDS}

active strain formulation, cardiac mechanics, finite element method, transmurally heterogeneous orthotropic activation

\section{1 | INTRODUCTION}

From a mathematical perspective, the contraction of the heart is a complex multiphysics and multiscale process. ${ }^{1}$ An electric signal spreads through the muscle, initiating subcellular chemical reactions and thus leading to cellular shortening. The synchronous shortening of the cardiac cells generates muscle contraction, which results in the ejection of blood from the atria to the ventricles and from the ventricles to pulmonary and systemic circulations. Any dysfunction in this sequence of events may have fatal consequences. ${ }^{*}$

Computational models can significantly help to increase the understanding of the heart function and dysfunction. As the computing power increases, electromechanical and electro-fluid-mechanical models of the heart have been developed and numerically solved. ${ }^{1,3-10}$ Unfortunately, the real predictive capabilities of such models are not always clear. In fact, many models fail in capturing the most obvious characteristics of the heart deformation. The left ventricular apex-to-base shortening, due to the downward motion of the aortic valve ring during systole, is the most important feature of cardiac contraction, indeed allowing the heart to work efficiently. Such deformation is often missing even in recent heart models. ${ }^{11}$ In some instances, the models may even yield an opposite behavior of what expected, thus resulting in apex-to-base

*In the United States of America, only the lifetime risk of developing heart failure is $20 \%$ for people over the age of 40 , with a $50 \%$ rate of mortality within 5 years of diagnosis. ${ }^{2}$ 
lengthening and unrealistic rotations..$^{12}$ This shows why the mathematical modeling of the active contraction of the heart is particularly challenging. Since the ventricular pressure-volume relations can be captured even in models where the ventricular wall motion is not accurately represented, the model validation requires a more in-depth comparison of the myocardial wall strains with respect to experiments.

Since the computational cost of multiphysics computational models is very large, new and enhanced mathematical descriptions of the cardiac contraction can be achieved more easily by better focusing on the outputs of interest. Hence, in this work, suitable assumptions are made to decouple the mechanical deformation of the ventricle from the fluid dynamics and from the electrophysiology. The influence of blood flow is accounted for by means of pressure boundary conditions (the force exerted on the endocardium), the electrical activation is assumed to be uniform in space, and the microscopic forces generated by the subcellular crossbridge dynamics are replaced by an explicit time-dependent function.

Typical models of passive mechanics are based on a hyperelastic assumption for the elastic behavior: The stress-strain relationship can be derived from the Helmholtz free energy characterizing the system. The myocardium is typically considered an orthotropic material ${ }^{13}$ or a transversely isotropic material. ${ }^{14}$ For the active contraction, two different approaches are commonly used: the active stress ${ }^{14-17}$ approach and the active strain approach. ${ }^{18-21}$ The former is based on an additive decomposition of the stress tensor into passive and active components, the latter instead on a multiplicative decomposition of the deformation gradient tensor. Actually, a formulation where the stress is additively decomposed into passive and active components can also be obtained by using a multiplicative decomposition of the deformation gradient tensor. ${ }^{22,23}$ In this sense, an active stress formulation can be obtained using an active strain approach. For historical reasons, we call active stress formulations only those where the decomposition of the deformation gradient tensor is not considered. Conversely, in an active strain formulation, the multiplicative decomposition of the deformation gradient tensor is applied to the whole free energy. These approaches to model the active contraction of the ventricular muscle can be derived from thermodynamical principles ${ }^{20}$ by postulating a special form of the free energy density function. In particular, it is assumed that the free energy depends on the deformation gradient tensor and on some internal variables describing the active state of the muscle. In the active stress models, the active component of the free energy is typically a function of a scalar internal variable, usually representing the biochemical reactions that lead to cellular contraction. ${ }^{20}$ In the active strain models, the internal variable describing muscular contraction is the active part of the deformation gradient tensor.

Two active strain models are present in literature: the transversely isotropic model ${ }^{19,24}$ and the orthotropic model. ${ }^{20}$ The former is unsuitable to yield physiological results in term of ejection fraction and wall thickening with a physiological fiber contraction. The latter, while providing physiological cardiac function when considering physiological fiber contraction of about $10 \%$, fails to reproduce physiological strain distribution. In particular, the strains in cross-fiber direction largely vary from less than $5 \%$ at the epicardium to more than $25 \%$ at the endocardium. ${ }^{25}$

In this work, we hypothesize that the ventricular cross-fiber strains observed experimentally ${ }^{25}$ are caused by a rearrangement of the cardiomyocytes within the collagen and that this rearrangement varies transmurally. We model the fibers rearrangement using an active strain formulation, which easily allows to model the anisotropic mechanical activation of the myocardium. The resulting model, the novel transmurally heterogeneous orthotropic model, is able to improve the prediction of the strains of current mechanical models of the left ventricle. The proposed model is well suited to describe physiological cardiac function with single myocyte contraction within the physiological range.

We compare the new model with experimental data and measurements, a recent active stress formulation ${ }^{17}$ and other active strain models. ${ }^{19,26}$ In particular, in our comparison, we consider the aforementioned transversely isotropic active strain model ${ }^{19,24}$ and the orthotropic activation model. ${ }^{20}$

In our computational model, which employs spatial approximation based on the finite element method, ${ }^{27}$ the equilibrium configuration of the system is described by the quasi-static formulation of the momentum equation. The constraint of incompressibility of the cardiac tissue is enforced using a penalty method, postulating the existence of a volumetric energy with a large bulk modulus. ${ }^{28}$ To reduce volumetric locking in the displacement-based formulation of the problem, we use a high-order finite element discretization of the quasi-static problem. ${ }^{29}$ In particular, we employ quadratic basis functions on simplex elements.

The paper is structured as follows. In Section 2, we introduce the models of the passive mechanics of the left ventricle. In particular, after recalling some notions of finite elasticity, we describe our choice of the constitutive model; we also describe the approach used to enforce the incompressibility constraint. In Section 3, we introduce the active strain approach and some of its variants. In Section 4, we derive the weak formulation of the quasi-static elasticity problem, and we describe the finite element formulation and the Newton method used to solve the nonlinear problem. In Section 5, we show the numerical results. We analyze the influence of the boundary conditions on the solution, and we compare our 
results against experimental measurements. By using this analysis, we select suitable boundary conditions for the comparison of the different activation models described in Section 3. By comparing again the numerical results with respect to experimental data at systole, we analyze which models better describe the ventricular contraction. We conclude in Section 6 by providing some perspective on the work done.

\section{PASSIVE CARDIAC MECHANICS}

In this section, we formulate the passive mechanical problem. We explain the assumptions at the basis of our formulation, and we describe the constitutive model.

\section{1 | Notion of continuum mechanics}

For a deforming body of material, we denote with $\Omega_{0}$ and $\Omega$ the initial and current configurations, respectively. In particular, we take $\Omega_{0}$ and $\Omega$ to be two open sets in $\mathbf{R}^{3}$ with Lipschitz boundaries. The deformation is characterized by the motion $\phi: \Omega_{0} \rightarrow \Omega$ such that $\mathbf{x}=\phi\left(\mathbf{x}_{0}\right)$, which maps the reference coordinate $\mathbf{x}_{0} \in \Omega_{0}$ to the current one $\mathbf{x} \in \Omega$. The vector $\mathbf{u}=\mathbf{x}-\mathbf{x}_{0}$ represents the displacement field and relates the position of a particle in the reference configuration $\mathbf{x}_{0}$ to its position in the current configuration $\mathbf{x}$. We denote the boundaries of $\Omega_{0}$ and $\Omega$ as $\Gamma_{0}=\partial \Omega_{0}$ and $\Gamma=\partial \Omega$, respectively. We also assume that $\boldsymbol{\phi}$ is a diffeomorphism from $\Omega_{0}$ to $\Omega$, such that the deformation gradient tensor $\mathbf{F}=\nabla \mathbf{x}=\mathbf{I}+\nabla \mathbf{u}$ and the deformation Jacobian determinant $J=\operatorname{det}(\mathbf{F})>0$ are always well defined. We denote with $\mathbf{H}=J \mathbf{F}^{-T}$ the cofactor of the tensor $\mathbf{F}$.

The equations of Lagrangian solid dynamics describe the rate of change of position, density, and momentum of a material body and can be expressed in an inertial reference frame. In the reference configuration, the equation of static equilibrium are expressed as

$$
\begin{gathered}
\rho J=\rho_{0} \quad \text { in } \Omega_{0}, \\
-\nabla_{0} \cdot \mathbf{P}=\rho_{0} \mathbf{b} \quad \text { in } \Omega_{0},
\end{gathered}
$$

where $\rho_{0}$ and $\rho$ are the reference and current densities, $\mathbf{P}\left(\mathbf{F}\left(\mathbf{x}_{0}\right)\right)$ is the first Piola-Kirchhoff stress tensor, $\nabla_{0}$. is the divergence operator with respect to the reference coordinates, and $\mathbf{b}$ represents the external forces acting on the body. In the theory of hyperelasticity, $\mathbf{P}$ is obtained from a strain energy function $\mathcal{W}(\mathbf{C})$ as

$$
\mathbf{P}=\frac{\partial \mathcal{W}}{\partial \mathbf{C}} \frac{\partial \mathbf{C}}{\partial \mathbf{F}}, \quad \mathbf{C}=\mathbf{F}^{T} \mathbf{F},
$$

where $\mathbf{C}$ is the right Cauchy-Green deformation tensor. Following the work of Nash and Hunter ${ }^{30}$ and Nash and Panfilov, ${ }^{31}$ we neglect the external and inertial forces. Equation $1 \mathrm{~b}$ needs to be supplied with appropriate boundary conditions. With this aim, we split the boundary of the left ventricle into three subsets $\Gamma_{0, D}, \Gamma_{0, N}$, and $\Gamma_{0, R}$, such that $\partial \Omega_{0}=\Gamma_{0, D} \cup \Gamma_{0, N} \cup \Gamma_{0, R}$. These are shown in Figure 1. $\Gamma_{0, D}$ represents the left ventricular basal plane, where we typically impose a Dirichlet condition to anchor the ventricle in order to avoid rigid displacements and rotations. Different choices are possible, such as preventing any displacement and rotation in the whole basal plane or preventing motion in the innermost region of the basal ring. Our choice is to constrain only the motion along the direction normal to the basal surface.

The surface region $\Gamma_{0, R}$ indicates the epicardium, the outmost external surface of the myocardium. Here, to represent the interaction between the ventricle and the adherent tissues, we set a Robin condition, which introduces a linear relation between displacement and stress:

$$
\mathbf{P N}_{0}=-\mathbf{A u}+\boldsymbol{\beta} \text { on } \Gamma_{0, R},
$$

where $\mathbf{A}$ is a second-order tensor representing the stiffness of adherent tissues, $\boldsymbol{\beta}$ a stress vector, and $\mathbf{N}_{0}$ is the outward direction unit vector normal to the reference surface $\Gamma_{0}$. Note that, by setting both $\mathbf{A}$ and $\boldsymbol{\beta}$ to zero, we obtain the usual stress free condition. By taking $\boldsymbol{\beta}=\mathbf{0}$ and $\mathbf{A}=\alpha \mathbf{I}$, we look for a suitable value for the coefficient $\alpha$ in order to provide a physically meaningful representation of the cardiac contraction. A positive value of $\alpha$ introduces a stiffness that prevents rigid body translations and rotations.

Finally, the surface region $\Gamma_{0, N}$ represents the endocardium, the inner part of the myocardium where the muscle is in contact with blood inside the ventricular cavity. On this portion of the boundary, we set a natural boundary condition 


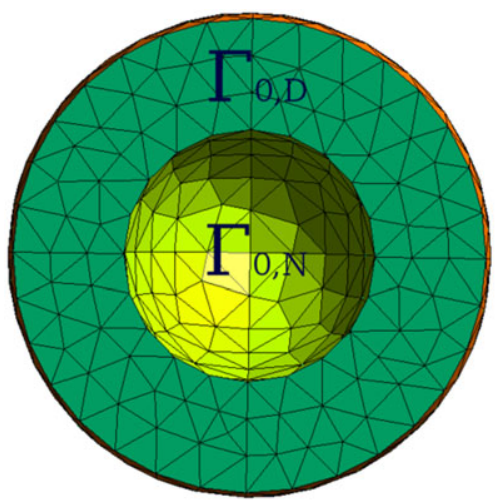

(A)

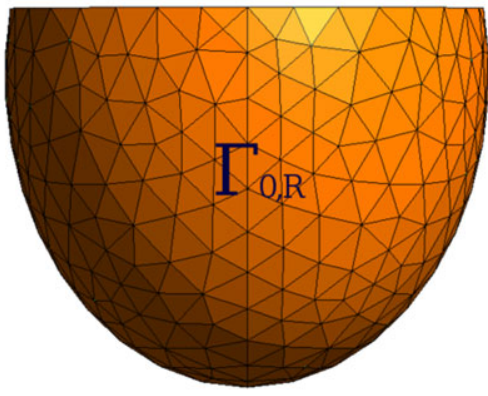

(B)

FIGURE 1 Idealized left ventricular geometry with boundaries $\Gamma_{0, D}, \Gamma_{0, N}, \Gamma_{0, R}$ highlighted in different colors to distinguish the three subsets of $\partial \Omega_{0}$ on which we set the boundary conditions (Dirichlet, natural, and Robin, respectively)

to describe the force that the blood exerts on the ventricular wall. We assume that the ventricular pressure in the cavity $p_{v}$ is uniformly distributed and that the stress is directed along the normal of the deformed surface. Since the deformed configuration is unknown, we resort to the Piola transformation, which is nonlinear, in order to reformulate the boundary condition in the known reference configuration:

$$
\mathbf{P N}_{0}=-p_{v} \mathbf{H} \mathbf{N}_{0} \quad \text { on } \Gamma_{0, N} .
$$

In summary, the final problem in its strong formulation, endowed with boundary conditions, reads:

$$
\begin{array}{rc}
-\nabla_{0} \cdot \mathbf{P}(\mathbf{u})=\mathbf{0} & \text { in } \Omega_{0}, \\
\mathbf{u} \cdot \mathbf{N}_{0}=\mathbf{0} & \text { on } \Gamma_{0, D}, \\
\left(\mathbf{I}-\mathbf{N}_{0} \otimes \mathbf{N}_{0}\right) \mathbf{P} \mathbf{N}_{0}=\mathbf{0} & \text { on } \Gamma_{0, D}, \\
\mathbf{P N}_{0}=-p_{v} \mathbf{H} \mathbf{N}_{0} & \text { on } \Gamma_{0, N}, \\
\mathbf{P N}_{0}=-\alpha \mathbf{u} & \text { on } \Gamma_{0, R} .
\end{array}
$$

All equations depend on the time $t>0$. Note that we omitted (1a) in (5) because incompressibility will be weakly enforced using a nearly incompressible formulation (see Section 2.3). By introducing the Hilbert space

$$
V=\left\{\mathbf{v} \in\left[H^{1}\left(\Omega_{0}\right)\right]^{3}: \mathbf{v} \cdot \mathbf{N}_{0}=0 \quad \text { on } \Gamma_{0, D}\right\},
$$

the weak formulation of problem (5) reads: Find $\mathbf{u} \in V$ such that

$$
\begin{aligned}
& \int_{\Omega_{0}} \mathbf{P}(\mathbf{u}): \nabla_{0} \mathbf{v d V}+\int_{\Gamma_{0, N}} p_{v} J \mathbf{F}^{-T} \mathbf{N}_{0} \cdot \mathbf{v d A}+ \\
& \int_{\Gamma_{0, R}} \alpha \mathbf{u} \cdot \mathbf{v d A}=0 \quad \forall \mathbf{v} \in V .
\end{aligned}
$$

The choice of the functional space is made compatibly with the need to compute gradient of $\mathbf{v}$ and $\mathbf{u}$ and to preserve square integrability in the sense of Lebesgue.

\section{2 | Constitutive law}

We still need to characterize, in problem (7), the form of the stress tensor. It is common to assume that the myocardial tissue is a hyperelastic material ${ }^{13,14,32,33}$ for which the stress tensor can be derived from a pseudo strain energy function, that representing cardiac tissue needs to account for the microstructural composition of the tissue, in which collagen sheets separate bundles of cardiac cells. ${ }^{34}$ We denote with $\mathbf{f}_{0}$ and $\mathbf{s}_{0}$ the orientation of the cardiac fibers and collagen sheets, 
respectively, and we assume that these two fields are mutually orthogonal, ie, $\mathbf{f}_{0} \cdot \mathbf{s}_{0}=0$. We create a local orthonormal frame of reference by introducing the cross-fiber field (normal to the collagen sheets) $\mathbf{n}_{0}=\mathbf{f}_{0} \times \mathbf{s}_{0}$. The orthonormal frame defined by the vectors $\left(\mathbf{f}_{0}, \mathbf{s}_{0}, \mathbf{n}_{0}\right)$ is local, as it follows the complex arrangement of the cardiac cells throughout the tissue. The vectors $\mathbf{f}, \mathbf{s}$, and $\mathbf{n}$ denote the deformed preferential directions $\mathbf{F f}_{0}, \mathbf{F s}_{0}$, and $\mathbf{F n}_{0}$, respectively. With the above assumptions, it is natural to consider the myocardial tissue as orthotropic; that is, the mechanical response of the tissue is different along the three mutually orthogonal direction $\left(\mathbf{f}_{0}, \mathbf{s}_{0}, \mathbf{n}_{0}\right)$. In this paper, we consider all these material properties by using the constitutive law proposed by Holzapfel and Ogden. ${ }^{13}$

To guarantee frame indifference, the strain energy function is defined as a function of the invariants of the right Cauchy-Green strain tensor $\mathbf{C}=\mathbf{F}^{T} \mathbf{F}$. In particular, the Holzapfel-Ogden constitutive law ${ }^{35}$ additively decomposes the soft isotropic mechanical response of the extracellular matrix and the stiff mechanical behavior of the fibers. By introducing the isotropic invariants

$$
\mathcal{I}_{1}(\mathbf{C})=\operatorname{tr} \mathbf{C}, \quad \mathcal{I}_{3}(\mathbf{C})=\operatorname{det} \mathbf{C}
$$

and the anisotropic invariants

$$
\begin{aligned}
& \mathcal{I}_{4, \mathrm{f}}(\mathbf{C})=\mathbf{f}_{0} \cdot \mathbf{C \mathbf { f } _ { 0 } ,}, \quad \mathcal{I}_{4, \mathrm{~s}}(\mathbf{C})=\mathbf{s}_{0} \cdot \mathbf{C} \mathbf{s}_{0}, \\
& \mathcal{I}_{8, \mathrm{fs}}(\mathbf{C})=\mathbf{f}_{0} \cdot \mathbf{C} \mathbf{s}_{0},
\end{aligned}
$$

this model is the sum of four independent terms

$$
\begin{aligned}
& \mathcal{W}(\mathbf{C})=\mathcal{W}\left(\mathcal{I}_{1}, \mathcal{I}_{4, \mathrm{f}}, \mathcal{I}_{4, \mathrm{~s}}, \mathcal{I}_{8, \mathrm{fs}}\right)=\mathcal{W}_{1}\left(\mathcal{I}_{1}\right)+ \\
& \mathcal{W}_{4, f}\left(\mathcal{I}_{4, \mathrm{f}}\right)+\mathcal{W}_{4, \mathrm{~s}}\left(\mathcal{I}_{4, \mathrm{~s}}\right)+\mathcal{W}_{8, f s}\left(\mathcal{I}_{8, \mathrm{fs}}\right),
\end{aligned}
$$

each component being of exponential type,

$$
\begin{aligned}
\mathcal{W}_{1}\left(\mathcal{I}_{1}\right) & =\frac{a}{2 b}\left(e^{b\left(\mathcal{I}_{1}-3\right)}-1\right), \\
\mathcal{W}_{4, f}\left(\mathcal{I}_{4, \mathrm{f}}\right) & =\frac{a_{\mathrm{f}}}{2 b_{\mathrm{f}}}\left(e^{b_{\mathrm{f}}\left(\left\{\mathcal{I}_{4, \mathrm{f}}-1\right\}\right)^{2}}-1\right), \\
\mathcal{W}_{4, \mathrm{~s}}\left(\mathcal{I}_{4, \mathrm{~s}}\right) & =\frac{a_{\mathrm{s}}}{2 b_{\mathrm{s}}}\left(e^{b_{\mathrm{s}}\left(\left\{\mathcal{I}_{4, \mathrm{~s}}-1\right\}\right)^{2}}-1\right), \\
\mathcal{W}_{8, f s}\left(\mathcal{I}_{8, \mathrm{fs}}\right) & =\frac{a_{\mathrm{fs}}}{2 b_{\mathrm{fs}}}\left(e^{b_{\mathrm{fs}} I_{8, \mathrm{fs}}^{2}}-1\right) .
\end{aligned}
$$

The symbol $\{\cdot\}$ denotes the Macaulay brackets. ${ }^{36}$

\section{3 | Nearly incompressible formulation}

Although the constitutive law (10) is written for an incompressible material, in order to account for the effects of blood perfusion in the tissue, we allow for small local volume changes adopting a nearly incompressible formulation for the left ventricular muscle. For that, we introduce a multiplicative decomposition of the deformation gradient tensor ${ }^{37}$

$$
\mathbf{F}=\overline{\mathbf{F}} \mathbf{F}_{\mathrm{vol}}
$$

that is, the local deformation is the sequential application of a purely volumetric deformation $\mathbf{F}_{\mathrm{vol}}$, followed by an isochoric one $\overline{\mathbf{F}}$. For an isotropic material, the multiplicative decomposition (12) yields an additive decomposition of the strain energy into a volumetric and isochoric term. Anisotropic materials require more caution. In fact, by applying (12) to the constitutive law (10), the response of the tissue under a spherical state of stress would be isotropic. Following Sansour ${ }^{38}$ and Helfenstein et al, ${ }^{39}$ we write (10) as

$$
\begin{aligned}
\mathcal{W}(\mathbf{C})= & \mathcal{W}_{\text {iso }}(\overline{\mathcal{I}})+ \\
& \mathcal{W}_{\mathrm{a}}\left(\mathcal{I}_{4, \mathrm{f}}, \mathcal{I}_{4, \mathrm{~s}}, \mathcal{I}_{8, \mathrm{fs}}\right)+\mathcal{W}_{\mathrm{vol}}\left(\mathcal{I}_{3}\right),
\end{aligned}
$$

where $\overline{\mathcal{I}}$ is the isotropic invariant of $\overline{\mathbf{C}}:=\overline{\mathbf{F}}^{T} \overline{\mathbf{F}}=J^{-\frac{2}{3}} \mathbf{C} . \mathcal{W}_{\text {iso }}$ defines the isotropic material behavior under isochoric motion, while $\mathcal{W}_{\mathrm{a}}$ contains the anisotropic response. The additional term $\mathcal{W}_{\text {vol }}$ is the energy term characterizing volume changes $J=\sqrt{I_{3}}$. It penalizes volume variations by making a deformation more energetically "expensive" when $J \neq 1$. 
Several expressions for $\mathcal{W}_{\mathrm{vol}}$ are proposed in literature ${ }^{40}$ : Generally, an optimal choice is to consider a penalty function bounded (from below), convex, and whose slope is null in $J=1$. In this work, we use the following ${ }^{41}$ :

$$
\mathcal{W}_{\mathrm{vol}}(J)=\frac{K}{4}\left(J^{2}-1-2 \ln J\right)
$$

Such volumetric energy is unbounded for $J \rightarrow 0$ or $J \rightarrow+\infty$ preventing the body to collapse into a point and to indefinitely dilate. The parameter $K$ in (14) is called bulk modulus. The bulk modulus is a penalization factor that allows to weakly enforce incompressibility. The final form of the stress tensor reads as follows:

$$
\begin{aligned}
\mathcal{P}= & a e^{b\left(\overline{\mathcal{I}}_{1}-3\right)} J^{-\frac{2}{3}}\left(\mathbf{F}-\frac{1}{3} \mathbf{F}^{-T}\right)+\frac{K}{2}\left(J^{2}-1\right) \mathbf{F}^{-T} \\
& +2 a_{\mathrm{f}}\left\{\mathcal{I}_{4, \mathrm{f}}-1\right\} e^{b_{\mathrm{f}}\left(\mathcal{I}_{4, \mathrm{f}}-1\right)^{2}} \mathbf{f} \otimes \mathbf{f}_{0} \\
& +2 a_{\mathrm{s}}\left\{\mathcal{I}_{4, \mathrm{~s}}-1\right\} e^{b_{\mathrm{s}}\left(\mathcal{I}_{4, \mathrm{~s}}-1\right)^{2}} \mathbf{s} \otimes \mathbf{s}_{0} \\
& +a_{\mathrm{fs}} \mathcal{I}_{8, \mathrm{fs}} e^{b_{\mathrm{fs}} I_{8, \mathrm{fs}}^{2}}\left(\mathbf{f} \otimes \mathbf{s}_{o}+\mathbf{s} \otimes \mathbf{f}_{0}\right)
\end{aligned}
$$

\section{3 | ACTIVE CARDIAC MECHANICS: THE ACTIVE STRAIN APPROACH}

Following the active strain model, ${ }^{18}$ we use a multiplicative decomposition of the deformation gradient tensor:

$$
\mathbf{F}=\mathbf{F}_{E} \mathbf{F}_{A},
$$

into an elastic deformation $\mathbf{F}_{E}$ and an active deformation $\mathbf{F}_{A}$. The idea behind this formulation is that an inelastic process, driven by a microscopic force generation mechanism led by electrophysiology, locally and actively modifies the length and the shape of the fibers; then, an elastic deformation accommodates such active strain distortion in order to preserve the compatibility. ${ }^{18}$ The physiological basis of this approach resides in the contractile units of the myocytes: $\mathbf{F}_{A}$ represents the sarcomeres shortening due to the sliding filaments of the actin-myosin molecular motor.

As explained in Ambrosi et al, ${ }^{18}$ the strain energy (13) only accounts for the elastic deformation; consequently, the Piola stress tensor is computed as

$$
\mathbf{P}_{E}=\frac{\partial \mathcal{W}}{\partial \mathbf{C}_{E}} \frac{\partial \mathbf{C}_{E}}{\partial \mathbf{F}_{E}}
$$

By pulling back to the reference configuration by means of the Piola transformation of $\mathbf{F}_{A}$, we have

$$
\mathbf{P}=\operatorname{det}\left(\mathbf{F}_{A}\right) \mathbf{P}_{E} \mathbf{F}_{A}^{-T}
$$

The expression of $\mathbf{F}_{A}$ must be provided according to a suitable constitutive law. Several descriptions of the active deformation $\mathbf{F}_{A}$ can be found in literature. ${ }^{19,20,24}$ In particular, we compare the transmurally heterogeneous orthotropic activation model with the transversely isotropic and the orthotropic activation models.

\section{1 | Transversely isotropic activation}

Since fibers contract along their axis, to enable fiber shortening and preserve microscopic volume changes, a transversely isotropic activation model can be considered. In fact, myocytes do not significantly change their volume during contraction. ${ }^{42}$ Therefore, a deformation along the fiber axis implies an isotropic deformation in the transverse plane to the fiber such that their volume is preserved. The active deformation gradient is assumed to take the form ${ }^{24,26}$

$$
\mathbf{F}_{A}=\gamma_{1} \mathbf{f}_{0} \otimes \mathbf{f}_{0}+\gamma_{2}\left(\mathbf{I}-\mathbf{f}_{0} \otimes \mathbf{f}_{0}\right)
$$

where the contraction $\gamma_{1}$ occurs along fibers direction $\mathbf{f}_{0}$, while $\gamma_{2}$ accounts for the deformation in the transverse directions. We assume in the following that $\operatorname{det}\left(\mathbf{F}_{A}\right)=1$; that is, the active deformations preserve volume of the myocyte. 
Specifically, for the transversely isotropic activation model, this condition reads

$$
\begin{aligned}
1 & =\operatorname{det}\left(\mathbf{F}_{A}\right)=\frac{\left(\mathbf{F}_{A} \mathbf{f}_{0} \times \mathbf{F}_{A} \mathbf{s}_{0}\right) \cdot \mathbf{F}_{A} \mathbf{n}_{0}}{\left(\mathbf{f}_{0} \times \mathbf{s}_{0}\right) \cdot \mathbf{n}_{0}} \\
& =\frac{\left(\gamma_{1} \mathbf{f}_{0} \times \gamma_{2} \mathbf{s}_{0}\right) \cdot \gamma_{2} \mathbf{n}_{0}}{\mathbf{n}_{0} \cdot \mathbf{n}_{0}}=\gamma_{1} \gamma_{2} \mathbf{n}_{0} \cdot \gamma_{2} \mathbf{n}_{0}=\gamma_{1} \gamma_{2}^{2},
\end{aligned}
$$

so that we can write $\gamma_{2}$ in terms of $\gamma_{1}$ explicitly

$$
\gamma_{2}=\frac{1}{\sqrt{\gamma_{1}}}
$$

Note that the requirement $\operatorname{det}\left(\mathbf{F}_{A}\right)=1$ is a constitutive assumption and this constraint is not namely required for the overall incompressibility of the tissue, which is instead assured by the nearly incompressible formulation. However, it allows to simplify the model, since in general, one must specify the evolution of the whole active deformation gradient $\mathbf{F}_{A} \cdot{ }^{11}$

By taking $\gamma_{1}=\gamma$, the final form of the active deformation gradient reads

$$
\mathbf{F}_{A}=\gamma \mathbf{f}_{0} \otimes \mathbf{f}_{0}+\frac{1}{\sqrt{\gamma}}\left(\mathbf{I}-\mathbf{f}_{0} \otimes \mathbf{f}_{0}\right)
$$

\section{2 | Orthotropic activation}

In order to capture the physiological systolic wall thickening, an alternative orthotropic model has been proposed in Rossi et al. ${ }^{20}$ The authors use a multiscale argument to link the microscale deformation occurring at the subcellular level with the macroscale deformation at the organ level. In particular, by assuming that the collagen fiber plays an active role during contraction and constraining the motion of the myocytes, the authors proposed an orthotropic activation model. The active deformation at microscale is isochoric, and it is described by means of the same transversely isotropic model described in Section 3.1. The active orthotropy originates from the inextensibility of collagen fibers that wrap around the cardiac cells: Contracting fibers enlarge in the directions orthogonal to their axis, and this enlargement forces the fibers to rearrange inside the collagen sheet, thus increasing the myocardial wall thickness. With this model, the myocardium can easily thicken up to $40 \%$ during contraction, ${ }^{43}$ and such thickening is assumed to be carried out in virtue of mutual sliding of collagen sheets. Moreover, the orthotropic model introduces an additional parameter that one can exploit to better describe the muscle thickening occurring during contraction.

The microscale active deformation is assumed to be in the form

$$
\begin{aligned}
\mathbf{F}_{M} & =\mathbf{I}+\xi_{f} \mathbf{f}_{0} \otimes \mathbf{f}_{0}+\xi_{s} \mathbf{s}_{0} \otimes \mathbf{s}_{0}+\xi_{n} \mathbf{n}_{0} \otimes \mathbf{n}_{0}, \\
\operatorname{det} \mathbf{F}_{M} & =\left(1+\xi_{f}\right)\left(1+\xi_{s}\right)\left(1+\xi_{n}\right)=1,
\end{aligned}
$$

where $\xi_{f}, \xi_{s}$, and $\xi_{n}$ are the microscopic deformations of fiber, sheet, and normal, respectively. The transverse isotropy requires that $\xi_{s}=\xi_{n}$ and the further assumption of isochoric deformation leads to $\xi_{n}=\left(1+\xi_{f}\right)^{-\frac{1}{2}}-1$. By introducing an intermediate-scale deformation where the cellular rearrangement takes place,

$$
\mathbf{F}_{S}=\mathbf{I}+\zeta_{S} \mathbf{s}_{0} \otimes \mathbf{s}_{0}+\zeta_{n} \mathbf{n}_{0} \otimes \mathbf{n}_{0}
$$

the macroscopic deformation tensor reads

$$
\mathbf{F}_{A}=\mathbf{F}_{S} \mathbf{F}_{M}=\mathbf{I}+\gamma_{f} \mathbf{f}_{0} \otimes \mathbf{f}_{0}+\gamma_{S} \mathbf{s}_{0} \otimes \mathbf{s}_{0}+\gamma_{n} \mathbf{n}_{0} \otimes \mathbf{n}_{0},
$$

where $\gamma_{j}=\xi_{j}+\zeta_{j}+\zeta_{j} \xi_{j}$, for $j=f, s, n$ represent deformations at the macroscale along fibers, sheets, and cross-fiber directions. By using multiscale arguments, Rossi et al ${ }^{20}$ relate the macroscopic deformations $\gamma_{\mathrm{f}}, \gamma_{\mathrm{s}}$, and $\gamma_{\mathrm{n}}$ with the microscopic shortening of a single cell $\xi_{f}$ such that

$$
\begin{gathered}
\gamma_{\mathrm{f}}\left(\xi_{f}\right)=\xi_{\mathrm{f}}, \\
\gamma_{\mathrm{n}}\left(\xi_{f}\right)=k \xi_{\mathrm{f}}, \\
\gamma_{\mathrm{s}}\left(\xi_{\mathrm{f}}\right)=\frac{1}{\left(1+\gamma_{\mathrm{f}}\right)\left(1+\gamma_{\mathrm{n}}\right)}-1,
\end{gathered}
$$


where $k$ is an orthotropic activation parameter that accounts for the collagen sheets rearrangement. The parameter $k$ above can be used to fit experimental data. As in Rossi et al, ${ }^{20}$ we set the parameter $k=4$. For more details on the derivation of the model, we refer the interested reader to the original paper. ${ }^{20}$

\subsection{Transmurally heterogeneous orthotropic activation}

Although the orthotropic model (Section 3.2) can easily capture the systolic wall thickening, the model fails to reproduce the heterogeneity of the deformation in the myocardium..$^{25}$ For this reason, starting from this orthotropic activation model, we develop a transmurally heterogeneous orthotropic activation model. Following Rossi et al, ${ }^{20}$ we extended their hypothesis of fiber rearrangement caused by the sheet sliding by including a transmurally varying rearrangement due to the tissue shearing induced by the torque exerted by the fibers at different transmural layers. The difference in torque is mainly due to different orientations, the different lever arms, and the difference in active forces due to the different passive stretch between fibers at different transmural layers. These differences result in a larger deformation in the inner layers of the myocardium caused by fibers rearrangement and resulting in increased wall thickness at the endocardium. This mechanical behavior is modeled by transmurally modulating the cross-fiber active deformation of the orthotropic active strain. More specifically, the cross-fiber active strain depends linearly on the transmural coordinate $\lambda$, where $\lambda$ is such that $\lambda=0$ at the endocardium while $\lambda=1$ at the epicardium. The transmural distance $\lambda$ is obtained using the Poisson interpolation, similarly to what presented in Rossi et $\mathrm{al}^{20}$ and Wong and $\mathrm{Kuhl} \mathrm{l}^{44}$ to prescribe fibers field. This parameter is used to locally adapt the rearranging mechanism from the endocardium, where the rearrangement occurs, to the epicardium, where the rearrangement is assumed to be negligible.

We notice that, as in Rossi et al, ${ }^{20}$ fibers at the microscale level are equally described by the isotropic active model (21) and share the same material properties throughout the whole ventricle. The main difference with the orthotropic model is the way fibers rearrange in the myocardium, which is mostly related to the overall behavior of the myocardium as a complex multiscale material. Following the multiscale argument that fibers rearrange within the collagen sheet ${ }^{20}$ and by including the transmural parameter inside (25), we derive the transmurally heterogeneous orthotropic model as follows:

$$
\begin{gathered}
\gamma_{\mathrm{f}}\left(\xi_{\mathrm{f}}\right)=\xi_{\mathrm{f}}, \\
\gamma_{n}\left(\lambda, \xi_{\mathrm{f}}\right)=(1-\lambda) k \xi_{\mathrm{f}}+\lambda \xi_{\mathrm{n}}, \\
=(1-\lambda) k \xi_{\mathrm{f}}+\lambda\left(\left(1-\xi_{\mathrm{f}}\right)^{-\frac{1}{2}}-1\right), \\
\gamma_{\mathrm{s}}\left(\lambda, \xi_{\mathrm{f}}\right)=\frac{1}{\left(1+\xi_{\mathrm{f}}\right)\left(1+\gamma_{\mathrm{n}}\left(\lambda, \xi_{\mathrm{f}}\right)\right)}-1 .
\end{gathered}
$$

In the following, we set $k=5.5$ uniformly in $\Omega_{0}$ in order to obtain the same ejection fraction of the orthotropic active strain model.

Remark 1. At the endocardium $(\lambda=0)$, we have

$$
\begin{gathered}
\gamma_{\mathrm{f}}\left(\xi_{\mathrm{f}}\right)=\xi_{\mathrm{f}}, \\
\gamma_{\mathrm{n}}\left(\lambda, \xi_{\mathrm{f}}\right)=k \xi_{\mathrm{f}}, \\
\gamma_{\mathrm{s}}\left(\lambda, \xi_{\mathrm{f}}\right)=\frac{1}{\left(1+\xi_{\mathrm{f}}\right)\left(1+k \xi_{\mathrm{f}}\right)}-1,
\end{gathered}
$$

coherently with (25).

\section{4 | NUMERICAL APPROXIMATION}

\section{1 | Spatial discretization}

The computational domain $\Omega_{0}$ we use in our test is based on an idealized geometry for the ventricle described by half of a prolate ellipse. ${ }^{16}$ The mesh is built from this geometry using linear tetrahedra, and the meshing process has been carried out using Gmsh. ${ }^{45}$ The meshing procedure introduces a partitioning $\mathcal{T}_{h}$ of the domain $\bar{\Omega}_{0}$ of nonoverlapping elements such that $\Omega_{0, h}=\cup_{K \in \mathcal{T}_{h}} K$. 
We choose the subspace $V_{h}$ of $V$ to be the finite element space of basis functions globally continuous and defined by Lagrange polynomials on each mesh element, ie,

$$
\begin{aligned}
V_{h}= & \left\{\mathbf{v}_{h} \in\left[C^{0}\left(\bar{\Omega}_{0, h}\right)\right]^{3}:\left.\left(\mathbf{v}_{h}\right)_{i}\right|_{K} \in \mathbb{P}_{r} \forall i=1,2,3,\right. \\
& \left.\forall K \in \mathcal{T}_{h}, \mathbf{v}_{h} \cdot \mathbf{N}_{0}=0 \text { on } \Gamma_{0, D}\right\} .
\end{aligned}
$$

In this work, we are interested in an accurate representation of strains, which are related to the gradient of the solution $\nabla_{0} \mathbf{v}_{h}$. By using piecewise linear polynomials $(r=1)$, this would result in piecewise constant strain functions, thus yielding at most first-order accuracy. Instead, using the finite element space with piecewise polynomials of degree $r=2$ improves the accuracy of the results. The quadratic representation induced by the choice of $r=2$ also improves the description of the fibers field, leading to a better characterization of the material properties of the myocardium.

A general function $\mathbf{v}_{h}$ of $V_{h}$ can be expressed as a linear combination of basis functions of (28)

$$
\mathbf{v}_{h}(\boldsymbol{\xi})=\sum_{i=1}^{M} v_{i} \boldsymbol{\phi}^{j}(\boldsymbol{\xi}),
$$

where $\xi \in \Omega_{0}, M$ is the total number of degrees of freedom introduced by the spatial discretization, and $\boldsymbol{\phi}^{i}$ is the $i$ th Lagrangian basis function. In this context, the displacement and the deformation gradient can be defined as

$$
\begin{gathered}
\mathbf{u}_{h}=\sum_{j=1}^{M} u_{j} \boldsymbol{\phi}^{i}(\boldsymbol{\xi}), \\
\mathbf{F}_{h}(\boldsymbol{\xi})=\mathbf{I}+\sum_{j=1}^{M} u_{j} \nabla_{0} \boldsymbol{\phi}^{j}(\boldsymbol{\xi}),
\end{gathered}
$$

respectively. Then, we define $J_{h}=\operatorname{det}\left(\mathbf{F}_{h}\right)$ and $\mathbf{H}_{h}=J_{h} F_{h}^{-T}$. By introducing Gauss-Legendre quadrature rules with $N_{Q p t}$ and $N_{B D Q p t}$ nodes over domain and boundaries, whose weights are $w\left(\xi_{q}\right)$ and $w_{B D}\left(\xi_{q}\right)$, respectively, and by plugging (30) and (31) into (7), we derive the corresponding algebraic formulation for the elastostatic problem. By setting

$$
\begin{gathered}
L_{i}\left(\mathbf{u}_{h}\right)=\sum_{q=1}^{N_{Q p t}} w\left(\boldsymbol{\xi}_{q}\right)\left[\mathbf{P}\left(\mathbf{F}_{h}\left(\boldsymbol{\xi}_{q}\right)\right): \nabla_{0} \boldsymbol{\phi}^{i}\left(\boldsymbol{\xi}_{q}\right)\right], \\
B_{i}^{N}\left(\mathbf{u}_{h}\right)=\sum_{q=1}^{N_{B D Q p t}} w_{B D}\left(\boldsymbol{\xi}_{q}\right)\left[p_{v} \mathbf{H}_{h}\left(\boldsymbol{\xi}_{q}\right) \mathbf{N}_{0} \cdot \boldsymbol{\phi}^{i}\left(\boldsymbol{\xi}_{q}\right)\right] \\
B_{i}^{R}\left(\mathbf{u}_{h}\right)=\sum_{q=1}^{N_{B D Q p t}} w_{B D}\left(\boldsymbol{\xi}_{q}\right)\left[\alpha \mathbf{u}_{h} \cdot \boldsymbol{\phi}^{i}\left(\boldsymbol{\xi}_{q}\right)\right]
\end{gathered}
$$

the sum of (32), (33), and (34) yields the algebraic nonlinear system

$$
\mathcal{L}\left(\mathbf{u}_{h}\right)=\mathbf{L}\left(\mathbf{u}_{h}\right)+\mathbf{B}^{N}\left(\mathbf{u}_{h}\right)+\mathbf{B}^{R}\left(\mathbf{u}_{h}\right)=\mathbf{0} .
$$

\subsection{Solution of the nonlinear problem}

We solve the nonlinear problem (35) by the Newton-Raphson method. ${ }^{46,47}$ The method exploits information from the Jacobian $J$ of $\mathcal{L}(\mathbf{u})$ to achieve the solution of the nonlinear problem:

$$
\begin{aligned}
& J_{i j}\left(\mathbf{u}_{h}\right) \delta u_{j}=\lim _{\epsilon \rightarrow 0} \frac{\mathcal{L}_{i}\left(\mathbf{u}_{h}+\varepsilon \delta \mathbf{u}_{j}\right)-\mathcal{L}_{i}\left(\mathbf{u}_{h}\right)}{\epsilon} \\
& =\sum_{q=1}^{N_{Q p t}} w_{q} \nabla_{0} \boldsymbol{\phi}_{q}^{i}: \frac{\partial \mathbf{P}}{\partial \mathbf{F}}\left(\mathbf{F}_{h}\left(\boldsymbol{\xi}_{q}\right)\right)\left[\delta u_{j} \nabla_{0} \boldsymbol{\phi}_{q}^{j}\right] \\
& \quad+\sum_{q=1}^{N_{B D Q p t}} w_{B D, q} \boldsymbol{\phi}_{q}^{i} \cdot\left[\alpha \delta u_{j} \boldsymbol{\phi}_{q}^{j}\right. \\
& \quad+\quad p_{v}\left[\left(\mathbf{F}_{h}^{-T}\left(\boldsymbol{\xi}_{q}\right): \delta u_{j} \nabla_{0} \boldsymbol{\phi}_{q}^{j}\right) \mathbf{I}\right. \\
& \left.\left.\quad-\mathbf{F}_{h}^{-T}\left(\boldsymbol{\xi}_{q}\right)\left(\delta u_{j} \nabla_{0} \boldsymbol{\phi}_{q}^{j}\right)^{T}\right] \mathbf{H}_{h}\left(\boldsymbol{\xi}_{q}\right) \mathbf{N}_{0}\right]
\end{aligned}
$$


where we denote with $\delta \mathbf{u}_{j}=\delta u_{k} \delta_{k, j}, w_{q}=w\left(\boldsymbol{\xi}_{q}\right), w_{B D, q}=w_{B D}\left(\boldsymbol{\xi}_{q}\right)$, and $\boldsymbol{\phi}_{q}^{j}=\boldsymbol{\phi}^{j}\left(\boldsymbol{\xi}_{q}\right)$. The fourth-order tensor $\partial \mathbf{P} / \partial \mathbf{F}$ depends only on the constitutive assumptions of the material, and it is computed from the strain energy density function as

$$
\frac{\partial \mathbf{P}}{\partial \mathbf{F}}=\frac{\partial^{2} \mathcal{W}}{\partial \mathbf{F} \partial \mathbf{F}}
$$

Given an initial guess $\mathbf{u}_{h}^{0}$, the solution of (35) is achieved as the limit (should it exist) of the sequence $\left\{\mathbf{u}_{h}^{n}\right\}$, such that

$$
\begin{gathered}
\mathbf{J}\left(\mathbf{u}_{h}^{n}\right) \delta \mathbf{u}^{n+1}=-\mathcal{L}\left(\mathbf{u}_{h}^{n}\right), \\
\mathbf{u}_{h}^{n+1}=\mathbf{u}_{h}^{n}-\delta \mathbf{u}^{n+1} .
\end{gathered}
$$

The stopping tolerance of the Newton method is set $10^{-7}$ over the $\infty$-norm of the residual $\mathcal{L}\left(\mathbf{u}_{h}^{n}\right)$.

Remark 2. Taking advantage of the flat surface at left ventricle base as seen in Figure 1A, with normal vectors aligned along one of the Cartesian axes, the Dirichlet boundary condition is enforced algebraically by manipulating the rows of the matrix:

$$
\begin{gathered}
J_{j_{D} j}=\delta_{j_{D}, j} \quad \text { for } j=1, . ., M, \\
\mathcal{L}_{j_{D}}=\delta \bar{u}_{j_{D}}^{n} \delta_{n, 0},
\end{gathered}
$$

being $j_{D}$ the index identifying a Dirichlet node and $\delta \bar{u}_{j_{D}}$ the value of the boundary condition.

Remark 3. The nonsymmetric linear system (36) is resolved using GMRES with an algebraic additive Schwarz preconditioner with local exact LU decompositions. ${ }^{48}$

\section{5 | NUMERICAL RESULTS}

We use a prolate ellipsoidal geometry fitted from the one used in Guccione et $\mathrm{al}^{16}$ by setting the same focal length of $2.91 \mathrm{~cm}$, cavity volume of $37 \mathrm{~mL}$, and a wall volume of $125 \mathrm{~mL}$. The geometry is described by the prolate ellipsoidal coordinates

$$
\begin{gathered}
x=d \sinh (\xi) \sin (\theta) \cos (\phi), \\
y=d \sinh (\xi) \sin (\theta) \sin (\phi), \\
z=d \cosh (\xi) \cos (\theta),
\end{gathered}
$$

where $d$ is the focal length, $\xi \in[0.6,1.02], \phi \in[0,2 \pi]$, and $\theta \in\left[\theta_{0}(\xi), \pi\right]$, with $\theta_{0}(\xi)$ such that the base is located at $z=1.19 \mathrm{~cm}$. Three meshes have been generated using mesh size of 1.0, 0.7, and $0.5 \mathrm{~cm}$, respectively. An example is shown in Figure 2. Since we compare strains resulting from our simulation with those reported in Guccione et al, ${ }^{16}$ we need to modify the constitutive parameters of our model in order to match those in Guccione et al. ${ }^{16}$ We therefore fitted

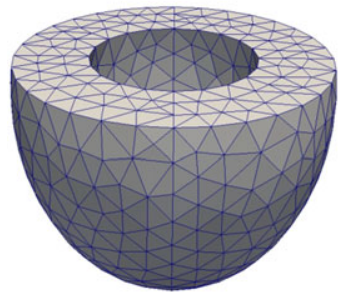

(A)

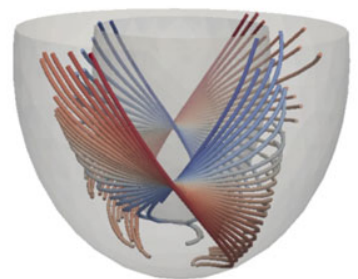

(B)

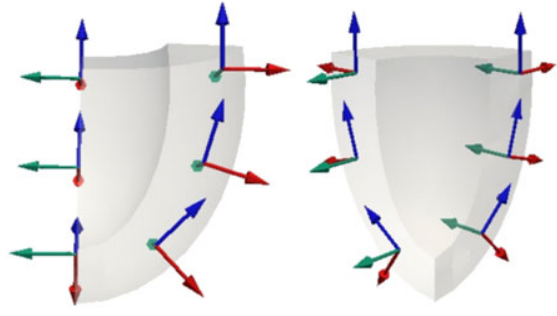

(C)

FIGURE 2 A, View of the mesh used for the strains analysis; the mesh comprises 668 vertices and 2380 tetrahedra. B, Transmurally symmetric distribution of the fibers; these are oriented with an helix angle ranging from $-60^{\circ}$ at the epicardium to $+60^{\circ}$ at the endocardium. C, Orthogonal triplets corresponding to the longitudinal (blue), radial (red), circumferential (green) directions (c); this shows also collagen sheets orientation since these are oriented along the radial direction 
TABLE 1 Parameters obtained from the least squares fitting of the Cauchy stress tensor

\begin{tabular}{llllllll}
$\boldsymbol{a}, \mathbf{k P a}$ & $\boldsymbol{b}$ & $\boldsymbol{a}_{\mathrm{f}}, \mathbf{k P a}$ & $\boldsymbol{b}_{\mathrm{f}}$ & $\boldsymbol{a}_{\mathbf{s}}, \mathbf{k P a}$ & $\boldsymbol{b}_{\mathbf{s}}$ & $\boldsymbol{a}_{\mathrm{fs}}, \mathbf{k P a}$ & $\boldsymbol{b}_{\mathrm{fs}}$ \\
\hline 0.20000 & 4.6140 & 4.1907 & 7.8565 & 2.5640 & 10.446 & 0.13040 & 15.255 \\
\hline
\end{tabular}

these parameters in our active strain implementation of the Holzapfel-Ogden constitutive law, to match the stress-strain relation of the Guccione law. ${ }^{16}$ To this purpose, we applied a least squares fitting strategy ${ }^{49}$ where we used as objective function the quadratic error between the six independent components of the Cauchy stress tensor obtained using the two constitutive laws. The result of the fitting is presented in Table 1.

The fiber and cross-fibers directions $\mathbf{f}_{0}$ and $\mathbf{n}_{0}$ are taken in an orthonormal system. We assume the collagen sheets to have a radial distribution. The fiber direction changes transmurally and linearly from $-60^{\circ}$ at the epicardium to $+60^{\circ}$ at the endocardium, while the cross-fiber direction goes from $+30^{\circ}$ at the epicardium to $-30^{\circ}$ at the endocardium. In our analysis, we consider the distribution of strains and shear strains in both space and time. In particular, by denoting with $\mathbf{c}, \mathbf{1}$, and $\mathbf{r}$ the circumferential, the longitudinal, and the radial directions, respectively, as shown in Figure $2 \mathrm{C}$, we define the following strains:

$$
\begin{array}{lll}
E_{c c}=\mathbf{E c} \cdot \mathbf{c}, & E_{l l}=\mathbf{E l} \cdot \mathbf{l}, & E_{r r}=\mathbf{E r} \cdot \mathbf{r}, \\
E_{c l}=\mathbf{E} \mathbf{c} \cdot \mathbf{1}, & E_{c r}=\mathbf{E c} \cdot \mathbf{r}, & E_{l r}=\mathbf{E} \mathbf{l} \cdot \mathbf{r},
\end{array}
$$

where $\mathbf{E}=\frac{1}{2}(\mathbf{C}-\mathbf{I})$ is the Green-Lagrange finite strain tensor. The above strains will be evaluated to compare our results with those in literature.

\section{1 | Convergence test under $h$-refinement}

We solve the problem with three isotropic meshes with mesh size $h=1.0,0.7$, and $0.5 \mathrm{~cm}$. We set the boundary conditions as in (5), with $\alpha=3.75 \mathrm{~mm} \mathrm{Hg} / \mathrm{cm}$ in (5e). We model the activation with the transmurally varying orthotropic model, with $k=4$. Each simulation consists of an entire cardiac cycle, which is divided in two main phases: diastole and systole. Each phase can be further split into a stage of null intraventricular flow rate (isochoric relaxation for diastole and isochoric contraction for systole) and a stage characterized by blood circulation (filling during diastole and ejection during systole). In this context, "isochoric" means that the deformation preserves the intraventricular volume. The four phases are governed by a relation between the cavity volume and the cavity pressure that can be represented in a pressure-volume loop diagram. In this test, the cycle starts with a preload ramp that inflates the ventricular cavity. This is achieved by setting increasing uniform pressure values on the endocardial surface until reaching the value $p_{m v c}$, the pressure at which the mitral valve closes. A system of idealized zero-dimensional valves is used to control the circulation of blood and prevents reverse flow due to pressure differences. The mitral valve allows flow entering the left ventricle whenever the ventricular preload $\left(p_{m v c}\right)$ is larger than the cavity pressure. Once the preload pressure is reached, the idealized mitral valve closes. At this stage, we initiate fibers activation by setting a uniform value for $\gamma_{\mathrm{f}}$ throughout the computational domain. The fibers start to contract determining the onset of the isochoric systolic phase. The evolution in time of the active strain is prescribed using the relation

$$
\gamma_{\mathrm{f}}(t)=\gamma_{\mathrm{f}, \min } \sin ^{2}\left(\pi \frac{t-t_{0}}{t_{F}-t_{0}}\right),
$$

where $t_{0}$ and $t_{F}$ are the initial and final times of the simulation and $\gamma_{f, \min }$ is the minimum value of the active strain (the largest in modulus). In this test, we set $\gamma_{\mathrm{f}, \min }=-0.13$. The evolution of the active strain $\gamma_{\mathrm{f}}$ is shown in Figure $3 \mathrm{~B}$.

During the isochoric phase, the ventricular pressure is unknown. Therefore, we look for a pressure value that keeps the cavity volume constant. With this aim, we use the fixed point iterative algorithm:

$$
\left\{\begin{array}{l}
C_{v}^{n}=V_{v}^{n} / p_{v}^{n} \\
p_{v}^{n+1}=p_{v}^{n}+\frac{\Delta V_{v}^{n}}{C_{v}^{n}}
\end{array}\right.
$$

where $n$ is the iteration number, $C_{v}^{n}$ is the ventricular compliance, $p_{v}^{n}$ is the cavity pressure, and $\Delta V^{n}=V^{n}-\bar{V}$ is the variation of the cavity volume. $\bar{V}$ is equal to the end-diastolic cavity volume during isochoric contraction and 


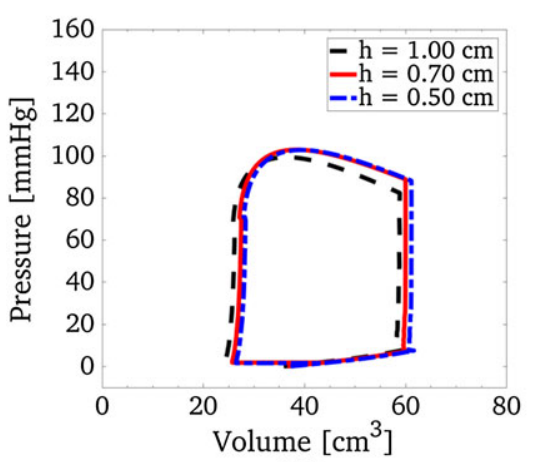

(A)

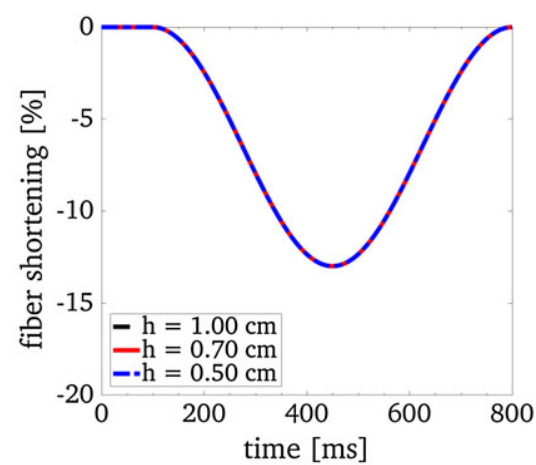

(B)

FIGURE 3 Simulations of a cardiac cycle using different mesh sizes $h=1.00,0.75$, and $0.50 \mathrm{~cm}$. In A, we represent the pressure-volume diagrams obtained with the three meshes. In B, we depict the evolution of the active strain activation $\gamma_{f}$ vs time

TABLE 2 Parameters used in simulations to regulate the cardiac cycle

$\begin{array}{lllll}\boldsymbol{p}_{\boldsymbol{m} v \boldsymbol{c}}, \mathbf{k P a} & \boldsymbol{p}_{\boldsymbol{a v o}}, \mathbf{k P a} & \boldsymbol{p}_{\boldsymbol{m} \boldsymbol{o}}, \mathbf{k P a} & \boldsymbol{R}_{\boldsymbol{w} \boldsymbol{k}}, \mathbf{k P a} \mathrm{ms} / \mathbf{m L} & \boldsymbol{C}_{\boldsymbol{w k}}, \mathbf{m L} / \mathbf{k P a} \\ 1 & 10 & 0.25 \mathrm{kPa} & 80000 & 0.00165\end{array}$

equal to end ejection cavity volume during isochoric relaxation. When the cavity pressure reaches the aortic pressure (initialized at $p_{A V O}$ ) at time $t_{B E}$, we set a dynamic afterload by coupling the cavity pressure with a two-element Windkessel model, ${ }^{50}$

$$
C_{w k} \frac{d p_{v}}{d t}=Q_{v}-p_{v} / R_{w k}, \quad t \in\left[t_{B E}, t_{E E}\right],
$$

where $t_{B E}, t_{E E}$ are time of begin ejection and time of end ejection, respectively, $p_{v}$ equals the aortic pressure as long as the aortic valve is open, $p_{v}\left(t_{B E}\right)$ is the pressure value at which the valve opens, and $Q_{v}$ is the outward flow rate from the ventricle. This phase continues until a positive value of $Q_{\nu}$ persists. As fibers start to relax, the flow rate becomes negative and the aortic valve closes, preventing the reversed flow and initiating the diastolic isochoric phase. Once again, the same isochoric process described before occurs, which is caused by the cavity pressure dropping below aortic one. The cavity volume does not change until a final pressure $\left(p_{m v o}\right)$ is achieved and the mitral valve opens, thus letting the blood to fill again the chamber. The simulation ends when fibers stop relaxing. The overall process can be observed in Figure 7B. Values used for this simulation are shown in Table 2. The overall procedure is summarized in Algorithm 1.

The results of the mesh convergence test under $h$-refinement are shown in Figures 3 and 4. Figure 3A shows the pressure-volume diagrams. We obtained three pressure-volume loops that mainly differ for the end-diastolic volume. From the coarsest mesh (black dashed line) to the finest one (dotted blue line), the maximum difference in terms of strain throughout the cardiac cycle decreases and we obtain that part of the intermediate mesh loop (solid red line) is superimposed on the blue line. Figure 4A-F reports the relation between strains and shear strains, averaged in the equatorial area of the ventricle, and the activation parameters $\gamma_{f}$ (in modulus), representing the active fiber shortening. This representation helps the interpretation of the results for its similarity with the pressure-volume diagram in Figure 3A since the positive correlation between $\left|\gamma_{f}\right|$ and the cavity pressure and the correlation between cavity volume and strains. The cardiac cycle starts with $\gamma_{f}=0$; then a horizontal segment determines the sign of the correlation between strains and $\left|\gamma_{f}\right|$ : positive for anticlockwise loops (Figure 4A,B,D,F) and negative for clockwise loops (Figure 4C,E), as indicated by the red arrow. The strains, except for Figure 4D, present a four-angle shape. Each angle represents a change in the cardiac phase. Starting from zero, the unloaded configuration, the initial horizontal segment corresponds to the passive inflation. Then, the ascending curve represents the systolic phase made of isovolumic contraction and ejection, and the descending part shows the diastolic phase made of isovolumic relaxation and filling. From Figure 4A-C, we see that the loops related to axial strains vary the most with the choice of the mesh. We deduce that the axial strains are more significantly affected by the discretization error than shear strains, in particular the circumferential strain, which varies of the $25 \%$ from the coarse to the finest mesh at both end-diastolic and end-systolic stages. The ventricle's average radius and length increase when inflated and the wall thickness decreases (as deduced from Figure 4A-C). These quantities remain approximately 


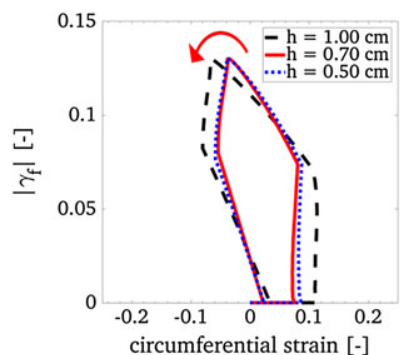

(A)

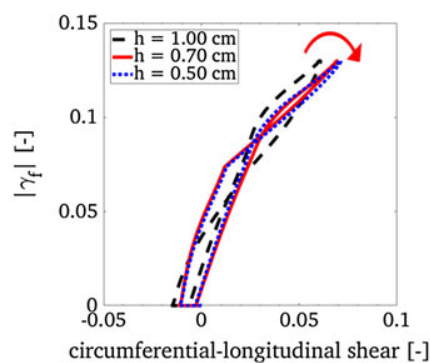

(D)

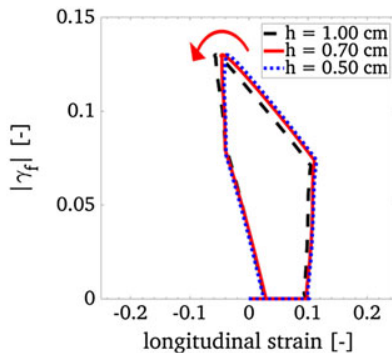

(B)

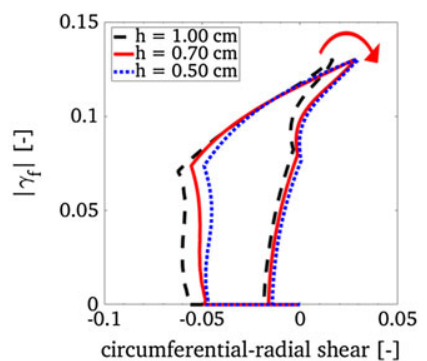

(E)

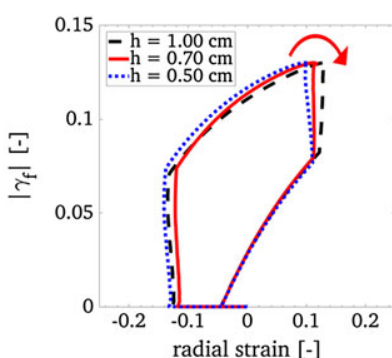

(C)

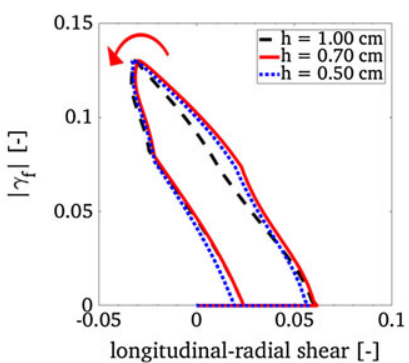

(F)

FIGURE 4 Simulations of a cardiac cycle using different mesh sizes $h=1.00,0.75$, and $0.50 \mathrm{~cm} . \gamma_{f}$ vs average equatorial strains (top) and shear strains (bottom)

constant during both isovolumic phases. During the ejection phase, the ventricular radius and its length both decrease, while the wall thickness increases to preserve the wall volume. Finally, during the relaxation phase, the ventricle returns to the initial unloaded configuration. The shear strains are depicted in Figure 4D-F. The circumferential-radial shear strain $E_{c r}$ describes the angle between the radial direction and the circumferential direction. This strain is closely related to the in-plane rotation, that is, the rotation around the apex-to-base axis. Figure 4E shows that this shear strain is always negative apart from a short time interval around end systole. Our interpretation is the following: When $E_{c r}$ is positive, $\cos \left(\theta_{r c}\right)$, being $\theta_{r c}$ the angle between the radial and the circumferential directions is also positive meaning that $\theta_{r c}$, which is $90^{\circ}$ in the reference configuration, decreases thus leading to a clockwise rotation around the apex-to-base axis. Conversely, a negative value of $E_{c r}$ describes a counterclockwise rotation. $E_{c l}$ (Figure 4D) describes the angle between the longitudinal and the circumferential directions and is related to ventricular torsion. Torsion can be explained as the in-plane rotation angle difference between two short axis sections at different ventricular height. With respect to the coordinate system shown in (Figure 2C), a positive circumferential-longitudinal shear strain $E_{c l}$ means that the in-plane rotation angle of the upper side of the ventricle is larger than the lower side, when considering positive any counterclockwise rotation around the apex-to-base apex. Conversely, the longitudinal-radial shear strain $E_{l r}$ is positive for almost the whole cardiac cycle apart between the last stage of the ejection and the initial stage of isovolumic relaxation when it becomes slightly negative. The interpretation of this shear is less straightforward. A positive value of this shear means that the angle between the deformed longitudinal direction and the deformed radial direction is an acute angle, thus inducing a tapered shape to the ventricle. A negative value of this shear strain means that the ventricle tends to assume a spherical shape.

From the plots in Figure 4, we observe that among the axial strains, $E_{c c}$ and $E_{r r}$ are the most affected by the choice of the mesh. For shear strains, noticeable differences can be observed when using the coarsest mesh, with $h=1 \mathrm{~cm}$. However, no significant differences are present between the intermediate mesh and the finest mesh, especially during end diastole and end systole considered in the following analysis. Therefore, hereafter, we use the intermediate mesh, with $h=0.7 \mathrm{~cm}$, which yields accurate results at a reduced computational cost.

\section{2 | Calibration of epicardial Robin coefficient $\alpha$ during passive inflation}

As stated in Section 2, we set Robin boundary conditions at the epicardium (see (5e)). The parameter $\alpha$ in the Robin condition is not know a priori. In order to choose it, we perform a passive inflation test of the left ventricle and we compare the strains obtained numerically against the experimental measurements in Omens et al. ${ }^{51}$ We finally select the value of $\alpha$ that better matches the experiments. 
We perform several numerical simulations using the same preload pressure $p_{m v c}$ of $1 \mathrm{kPa}$ for different values of the Robin coefficient $\alpha$ ranging from $75 \mathrm{~mm} \mathrm{Hg} / \mathrm{cm}$ (stiffer) to $0.75 \mathrm{~mm} \mathrm{Hg} / \mathrm{cm}$ (softer). In Figure 5, we show the three axial strains and the three shear strains: circumferential $\left(E_{c c}\right)$, longitudinal $\left(E_{l l}\right)$, radial $\left(E_{r r}\right)$, circumferential-longitudinal $\left(E_{c l}\right)$, circumferential-radial $\left(E_{c r}\right)$, and longitudinal-radial $\left(E_{l r}\right)$. We also show the experimental strain measurements from Omens et al ${ }^{51}$ depicted with bars indicating their standard deviation. The computationally evaluated strain is taken at equatorial height of the ventricle in agreement with the reference data.

As it can be seen in Figure 5A-C, the axial strains are in very good qualitative and quantitative agreement with the measurements when a small value of $\alpha$ is set. In particular, circumferential and radial strains converge within the experimental range by lowering the stiffness of the surrounding material, thus indicating that the myocardium itself is able to absorb the elastic energy by counterbalancing the cavity pressure with its stiffness. Conversely, the longitudinal strain $\left(E_{l l}\right)$, which during diastole describes the elongation of the ventricle, does not match within experimental range by lowering $\alpha$. As both $E_{l l}$ and the intraventricular volume are in agreement with experimental data, a possible explanation of this outcome is that the pericardium limits the compliance of the ventricle leading to a lower end-diastolic cavity volume on equal loading condition. This was also observed experimentally. ${ }^{52}$

Regarding shear strains, a qualitative agreement is obtained for circumferential-longitudinal $\left(E_{c l}\right)$ shear for every value $\alpha$ considered. For circumferential-radial $\left(E_{c r}\right)$ and longitudinal-radial $\left(E_{l r}\right)$ shears, the match is unsatisfactory for every choice of the parameter $\alpha$. Shear strains do not appear to be affected by Robin boundary condition at the epicardium, but rather by constitutive assumptions. For this reason, in this test, we do not take these data into account to calibrate the Robin coefficient. Following this analysis, we set $\alpha=7.5 \mathrm{~mm} \mathrm{Hg} / \mathrm{cm}$ in numerical simulations considering the qualitatively good agreement with experimental data in terms of axial strains and the physiological filling volume obtained in agreement with Omens et al. ${ }^{51}$

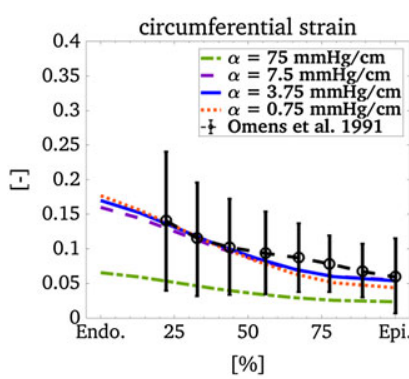

(A)

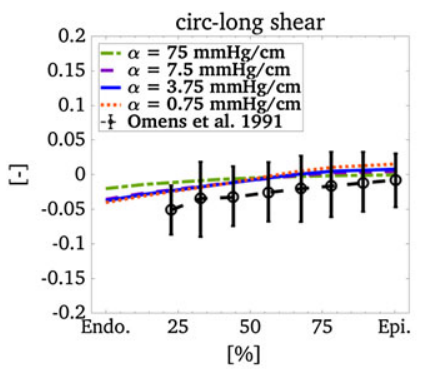

(D)

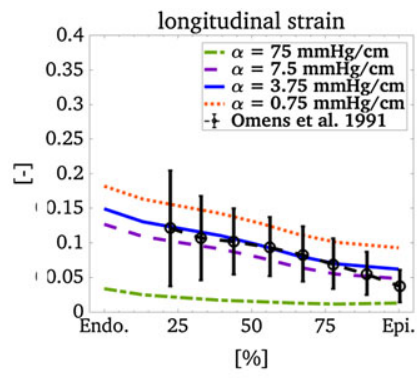

(B)

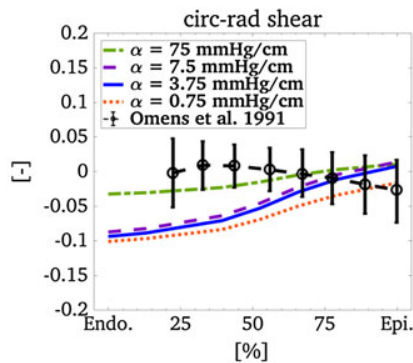

(E)

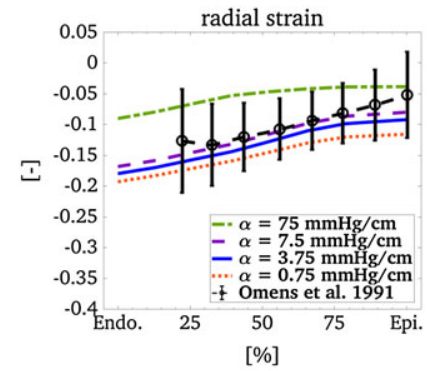

(C)

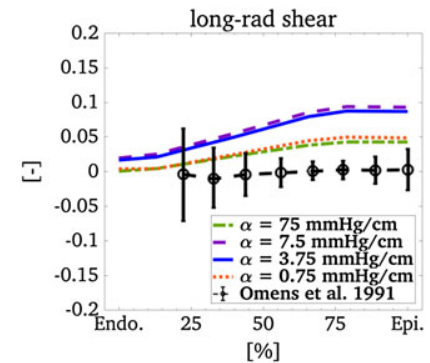

(F)

FIGURE 5 Comparison of the end-diastolic strains using different Robin coefficients for the epicardial boundary condition. Top row: axial strains (from left to right: $E_{c c}, E_{l l}$, and $E_{r r}$ ). Bottom row: shear strains (from left to right: $E_{c l}, E_{c r}$, and $E_{l r}$ ). Strains, both experimental and numerical, are measured through the myocardium at equatorial height. Strains are represented over wall thickness expressed in percentage. Experimental data are reported with error bars indicating standard deviations. The endocardial pressure was set to $1 \mathrm{kPa}(7.5 \mathrm{~mm} \mathrm{Hg}$ ) according to experiment in Omens et $\mathrm{al}^{51}$ 


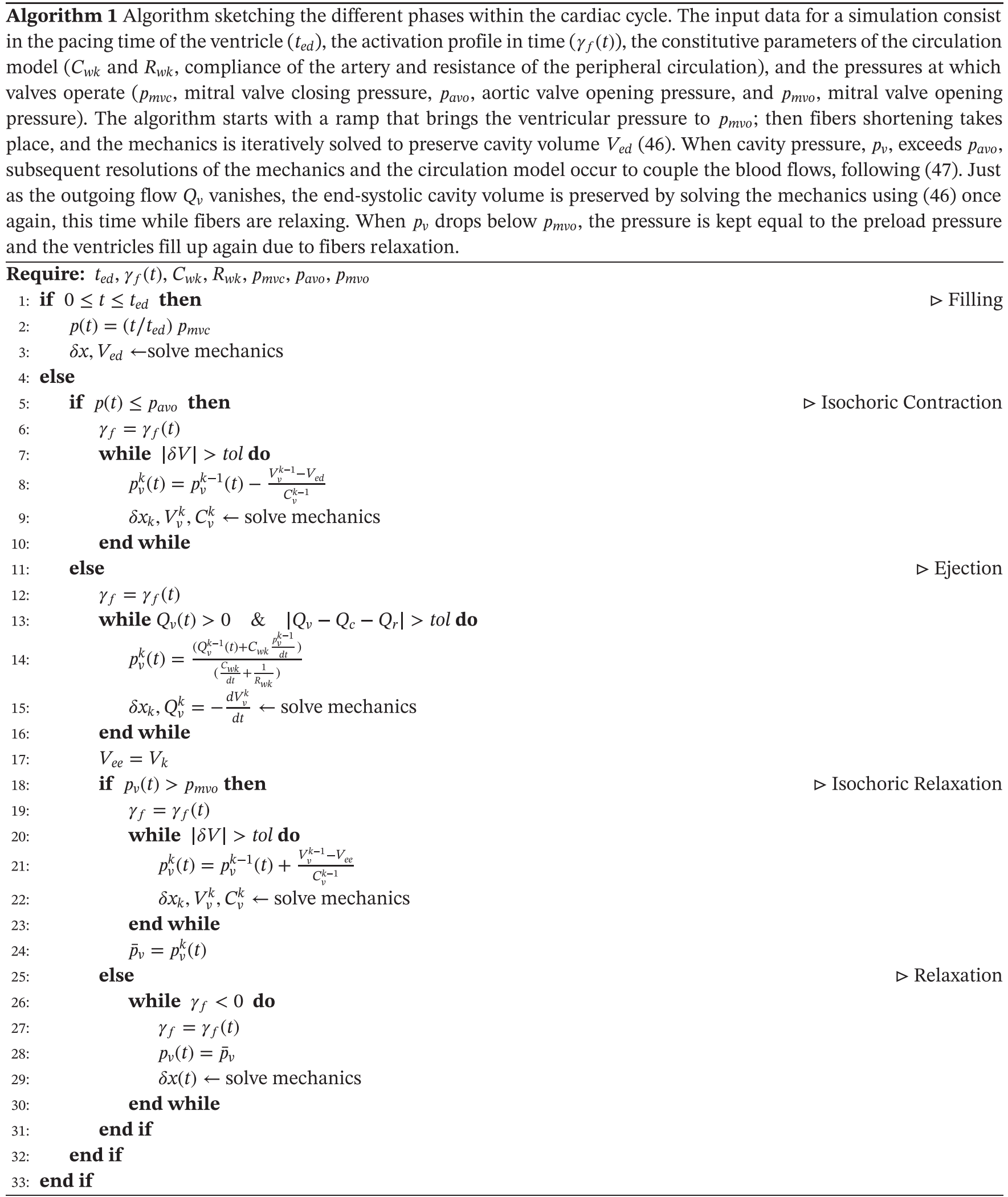

\section{3 | Effects of nonuniform activation}

So far, we assumed that the mechanical activation of myofibers is simultaneous throughout the myocardium. This is a simplification that we made in our model. As a matter of fact, myocardial mechanical activation originates from the electrical stimulus that presents local regional differences. However, these local regional electrical differences in physiological 
circumstances results into a time delay in the mechanical activation that amounts to approximately $50 \mathrm{~ms}^{53}$ Therefore, in order to better assess the validity of our assumption, we introduce a time delay in the mechanical activation and we compare the results with those with simultaneous and uniform mechanical activation. We made two simulations where a transmural and a longitudinal apex-to-base delay of $50 \mathrm{~ms}$ has been introduced, respectively. In these simulations, we used the transversely isotropic active strain model in which we set the fiber mechanical activation parameter, $\gamma_{f}$, as in (45). In the case of uniform activation, the same $\gamma_{f}$ is set everywhere in the myocardium. Instead, to model the transmural delay we included in (45), a transmural delay linearly varying from $0 \mathrm{~ms}$ at the endocardium to $50 \mathrm{~ms}$ at the epicardium, while the longitudinal delay varies from $0 \mathrm{~ms}$ at apex to $50 \mathrm{~ms}$ at base. Recalling (45), the delayed $\gamma_{f}$ is computed as follows:

$$
\left\{\begin{array}{c}
t_{a}(\mathbf{x})=\{t-50 \xi(\mathbf{x})\}, \\
\gamma_{\mathrm{f}}\left(t_{a}(\mathbf{x})\right)=\gamma_{\mathrm{f}, \min } \sin ^{2}\left(\pi \frac{t_{a}(\mathbf{x})-t_{0}}{t_{F}-t_{0}}\right),
\end{array}\right.
$$

where $t_{a}$ is the spatially varying mechanical activation time and $\xi(\mathbf{x})$ can be the transmural coordinate $\lambda(\mathbf{x})$ or the longitudinal coordinate $l(\mathbf{x})=\left(z(\mathbf{x})-z_{\text {apex }}\right) /\left(z_{\text {base }}-z_{\text {apex }}\right)$ for the transmural and longitudinal delay, respectively.

Figure 6A shows how such delays introduce a shift in the pressure profile of about $30 \mathrm{~ms}$ with respect to the case of uniform mechanical activation. However, the influence of both transmural and longitudinal delay is qualitatively less evident by looking at strains. Figure 6B-G shows the transmural distribution at midventricular height of the end-systolic strains at $14 \mathrm{kPa}$. The change in magnitude is very limited, and the transmural trend of all the strains remains unaffected by the introduction of the delays. Therefore, this clearly support our assumption of uniform mechanical activation for the transmural strain distribution.

\subsection{Comparison of active contraction models}

We compare the cardiac cycles for the three activation models described in Section 3. The cardiac cycle simulation has been discussed in Section 5.1, and it is summarized in Algorithm 1. Additionally, the Robin coefficient in Equation 5 is set to $\alpha=7.5 \mathrm{~mm} \mathrm{Hg} / \mathrm{cm}$. This choice, which we discussed in Section 5.2, allows us to better match the diastolic strains.

The evolution of the activation $\gamma_{f}$ is prescribed using Equation 45. The maximum active shortening $\left(\gamma_{f, \min }\right)$ is tuned in each model in order to obtain a similar ventricular ejection fraction. In this way, we compare the models under similar hemodynamics conditions. The $\gamma_{f}$ function is reported in Figure 7A for the three activation models. Here, we observe that, while for orthotropic models, the maximum active shortening lays inside a physiological range between -0.1 and

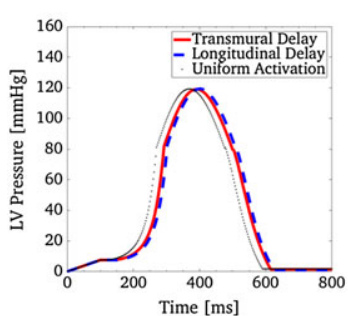

(A)

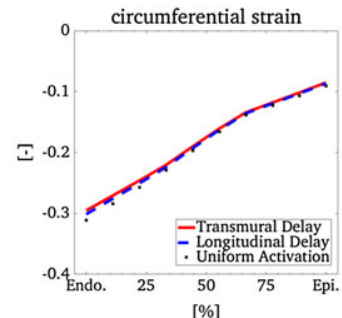

(B)

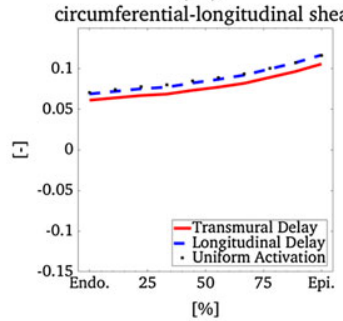

(E)

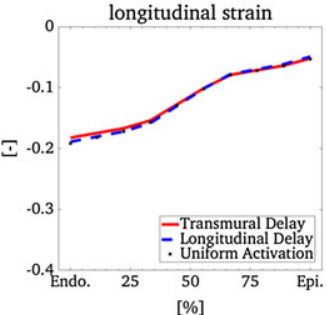

(C)

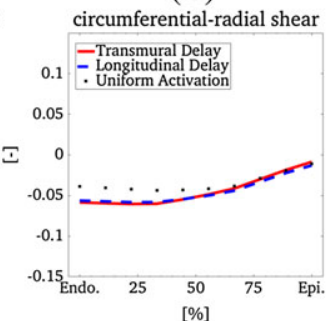

(F)

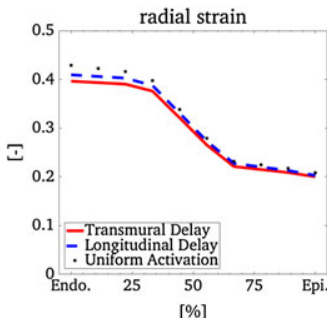

(D)

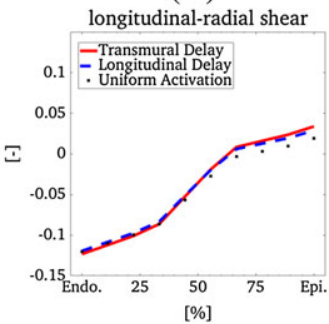

(G)

FIGURE 6 A, A comparison of the left ventricular pressure profiles obtained by setting uniform activation (black dotted), longitudinal delay (blue dashed line), and transmural delay (red solid line). B-G, A comparison of the transmural distribution of the end-systolic strains at cavity pressure $p_{v}=14 \mathrm{kPa}$ (approximately $105 \mathrm{~mm} \mathrm{Hg}$, reached between 430 and $462.5 \mathrm{~ms}$ ). The strains are evaluated at equatorial height and plotted vs the normalized transmural length. The three lines represent strains obtained using the transversely isotropic activation model using a uniform mechanical activation (black dotted), a 50-ms longitudinally delayed mechanical activation (blue dashed line), and a 50-ms transmurally delayed mechanical activation (red solid line) 


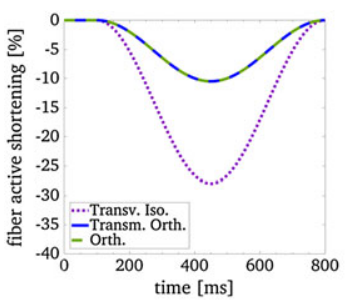

(A)

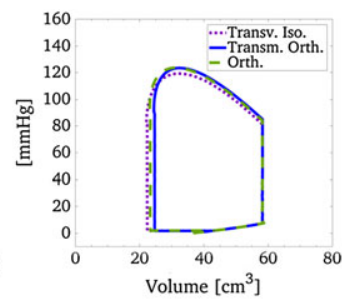

(B)

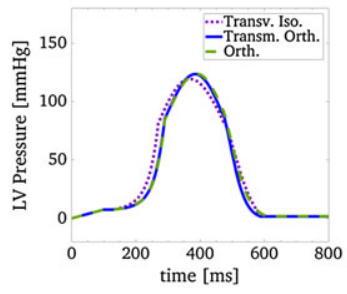

(C)

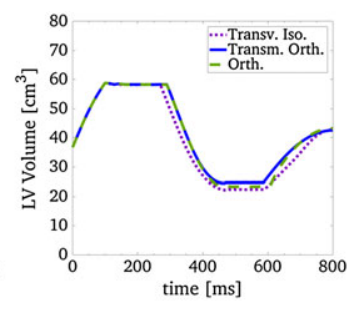

(D)

FIGURE 7 A, Time evolution of the active strain $\gamma_{f}$. B, Pressure-volume diagrams. C, Left ventricular cavity pressure. D, Left ventricular cavity volume throughout the cardiac cycle

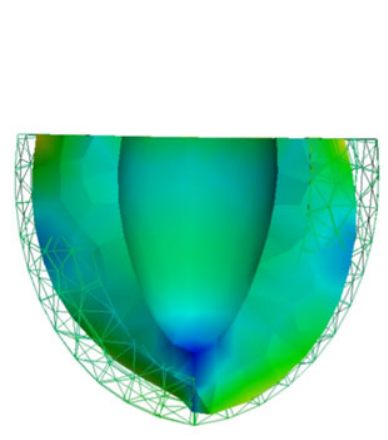

Transv. Iso.

(A)

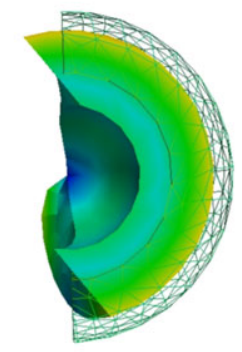

Transv. Iso.

(D)

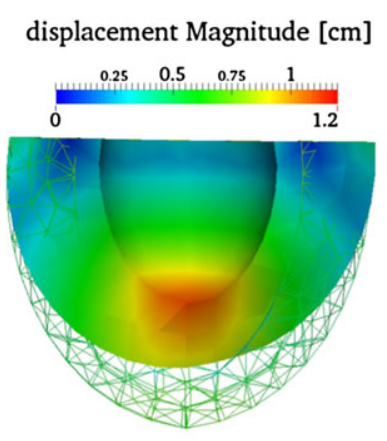

Orth.

(B)

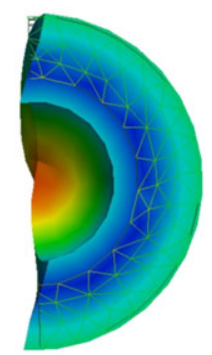

Orth.

(E)

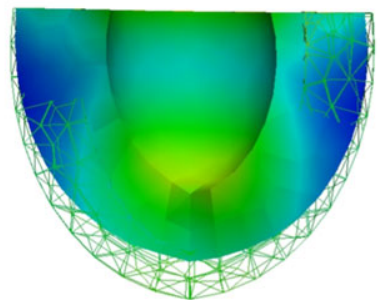

Transm. Orth.

(C)

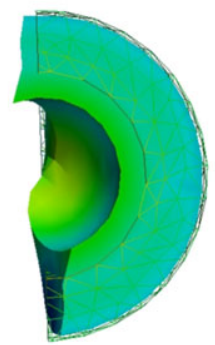

Transm. Orth.

(F)

FIGURE 8 Three-dimensional deformation of the three activation models at $p_{v}=14 \mathrm{kPa}$ over the end-diastolic configuration

-0.15 , for the transversely isotropic activated material, $\gamma_{f}$ lays outside this range. In particular, we set $\gamma_{f, \min }=-0.28$ for the transversely isotropic activation model and $\gamma_{f, \min }=-0.105$ for the orthotropic model and for the heterogenous orthotropic model, using an orthotropic activation parameter $k=4$ and $k=5.5$, respectively.

Figure 7B shows a comparison of the pressure-volume loops obtained with the three activation models under consideration. The three curves are close enough to enable comparisons of the strains at final systolic stage. Figure 7C,D suggests that the transversely isotropic model has a slightly different pressure development, most probably due to the larger increments in the fibers shortening that determine a different timing in the opening/closing of valves. Despite this small delay, the pressure-volume loop in Figure 7B shows that the end-systolic comparison at $14 \mathrm{kPa}$ can be made at almost same ejection fraction (about 60\%).

Figure 8 shows the deformed configuration in which we computed strains (shown in Figure 9) at $p_{v}=14 \mathrm{kPa}$ of cavity pressure. The top row shows the side view of a section of the idealized ventricle. The bottom row shows the deformed cavities and the basal planes. We show the transversely isotropic model, the orthotropic model, and the transmurally heterogeneous orthotropic model from left to right. The side view highlights the different behaviors of the two orthotropic models: They both exhibit a similar shortening and wall thickening, while the transversely isotropic model has a smaller ventricular shortening and a more elongated shape. The basal view highlights that the three models have different behaviors in terms of rotation (in-plane rotation) and torsion (rotation gradient: offset between basal and apical rotations). The transversely isotropic model and the transmurally heterogeneous orthotropic models have similar torsions but opposite 


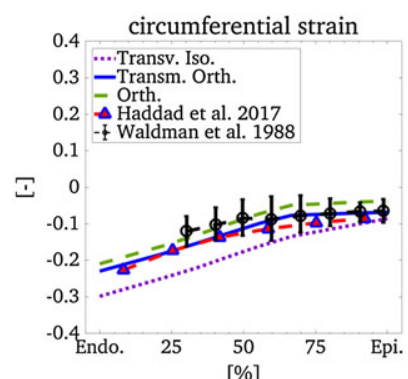

(A)

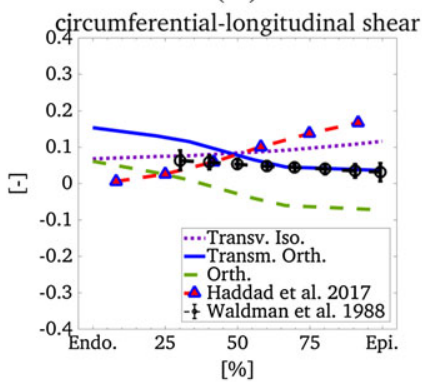

(D)

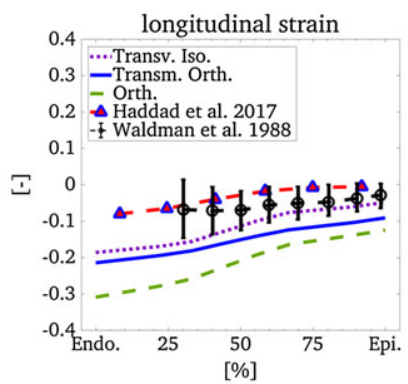

(B)

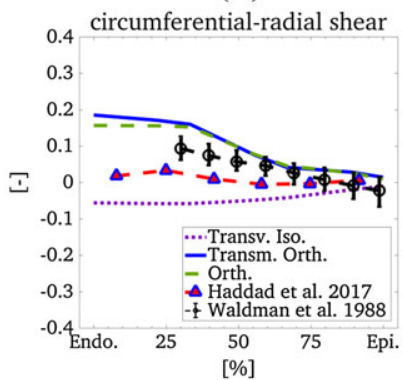

(E)

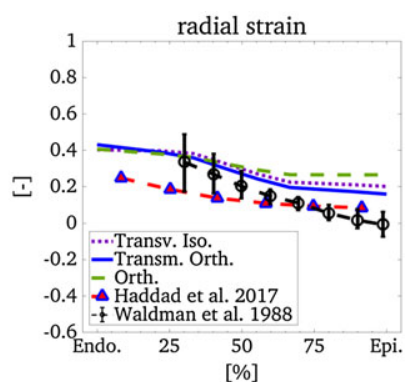

(C)

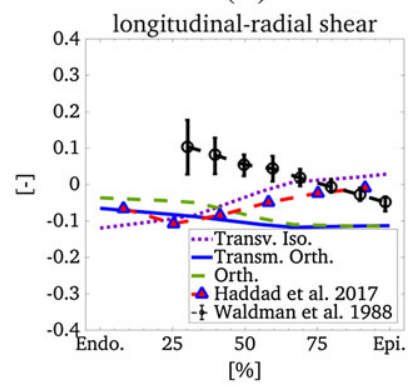

(F)

FIGURE 9 Comparison of the systolic strains at cavity pressure $p_{v}=14 \mathrm{kPa}$. The strains are evaluated at equatorial height and plotted vs the normalized transmural length. The three lines with no markers represent results stemming the transversely isotropic (.•), orthotropic (-), and transmurally heterogeneous orthotropic (-) active strain model. The marked line shows results using the active stress model of Haddad and Samani, ${ }^{17}$ the error bars, instead, experimental measurements from Omens et $\mathrm{al}^{51}$

in-plane rotations, suggesting that the two models should have similar values for the circumferential-longitudinal shear strains $E_{c l}$ but different circumferential-radial shear strain $E_{c r}$ (see Figure 9D,E). In fact, being the torsion related to the difference of in-plane rotation between slices at different ventricular height, it is possible to obtain the same torsion level with opposite in-plane rotations. For instance, taking two slices, one at base and one at apex, the same torsion can be obtained with two counterclockwise or two clockwise in-plane rotations provided that in both cases, the larger rotation (considering signs) occurs on the same slice. The orthotropic model exhibits both rotation and torsion reversed with respect to the transversely isotropic model. The comparison of shear strains with experimental data confirms that the correct behavior is the one featured by the transmurally orthotropic activation model (Figure $8 \mathrm{~F}$ ).

Starting from circumferential strains in Figure 9A, the two orthotropic models yield results close to the experimental data while the transversely isotropic activation has larger circumferential shortenings. As depicted in Figure 8A, this results in an excessive reduction of the cross-sectional area of the ventricular cavity. According to experimental data, this behavior seems to be unphysiological. By observing Figure 9B, the transversely isotropic activation provides longitudinal strains in qualitative agreement with the experiments for the longitudinal strains. As shown in Figure 8B, the shortening occurring in the orthotropic activation model is too large and resides outside experimental data range. The transmurally heterogeneous orthotropic activation model provides longitudinal strain slightly outside the experimental data range and qualitatively close to the one predicted by the transversely isotropic activation model. Considering radial strains $E_{r r}$, the three models well describe wall thickening at the endocardium. However, all of them fall outside the experimental range at the epicardium, even though the transmurally orthotropic activation model presents a slightly improved prediction. By checking the circumferential-longitudinal shear strain $E_{c l}$ against the experimental data in Figure 9D, we deduce which activation model is capable of correctly reproducing the ventricular torsion. The orthotropic models fail to properly represent torsion since provides torsion in the opposite direction. The transversely isotropic activation model instead, despite predicts torsion close to the experimental range, shows a reversed transmural distribution (strains increasing from the endocardium to the epicardium, instead of decreasing). This is also confirmed by the circumferential-radial shear strains-related closely to in-plane rotation-shown in Figure 9E, where the transversely isotropic model has opposite sign with respect to the experimental data. The transmurally heterogeneous orthotropic model proposed in this paper captures the ventricular torsion correctly. Finally, Figure $9 \mathrm{~F}$ shows that none of the models herein analyzed is able to provide a good qualitative match of the longitudinal-radial shear strains $E_{l r}$. One possible reason for this behavior is that longitudinal-radial shear strains $E_{l r}$ are affected by the orientation of the fiber field, which we maintained fixed for all the simulations. 
TABLE 3 Summary of the strain comparison between the analyzed models

\begin{tabular}{|c|c|c|c|c|c|c|c|c|c|c|}
\hline \multirow[b]{3}{*}{$E_{c c}$} & \multicolumn{2}{|c|}{ Transv. Iso } & \multicolumn{2}{|l|}{ Orth. } & \multicolumn{2}{|c|}{ Transm. Orth. } & \multicolumn{2}{|c|}{ Active Stress } & \multicolumn{2}{|c|}{ Exp. Measurements 5} \\
\hline & Trend & Error & Trend & Error & Trend & Error & Trend & Error & Trend & Error \\
\hline & $\nearrow$ & 0.0760 & $\nearrow$ & 0.0224 & $\nearrow$ & 0.0236 & $\nearrow$ & 0.0320 & $\nearrow$ & 0.0459 \\
\hline$E_{l l}$ & $\nearrow$ & 0.0504 & $\nearrow$ & 0.1385 & $\nearrow$ & 0.0842 & $\nearrow$ & 0.0347 & $\nearrow$ & 0.0539 \\
\hline$E_{r r}$ & $\searrow$ & 0.1219 & $\searrow$ & 0.1531 & $\searrow$ & 0.0930 & $\searrow$ & 0.0910 & $\searrow$ & 0.0832 \\
\hline$E_{c l}$ & $\nearrow$ & 0.0456 & $\searrow$ & 0.0895 & $\searrow$ & 0.0284 & $\nearrow$ & 0.0754 & $\searrow$ & 0.0181 \\
\hline$E_{c r}$ & $\nearrow$ & 0.0974 & $\searrow$ & 0.0406 & $\searrow$ & 0.0436 & $\searrow$ & 0.0466 & $\searrow$ & 0.0320 \\
\hline$E_{l r}$ & $\nearrow$ & 0.1020 & $\searrow$ & 0.1301 & $\searrow$ & 0.1490 & $\nearrow$ & 0.1174 & $\searrow$ & 0.0372 \\
\hline Average & & 0.0822 & & 0.0957 & & 0.0703 & & 0.0662 & & 0.0451 \\
\hline
\end{tabular}

The table reports the standard deviation of the experimental measurement error and for each model the transmural trend of each strain and the error with respect to the experimental measurement. The indicated error is the standard deviation along the line used in the comparison of Figure 9 between the difference between the strain predicted by the model and the mean value of the experimental strain measurement, computed according to (49). The error has been computed using the points in the region where the experimental data were available: from about $30 \%$ to about $90 \%$ of transmural depth. The transmural trend of the strain from the endocardium to the epicardium is written in black if it is in agreement with experimental data, in red otherwise. For each row, the largest error is written in red and the smallest one in green. In the last row, the average error over the strains is reported.

Additionally, we compare our results with those obtained for the left ventricular systolic strain using instead an active stress formulation. ${ }^{17}$ We represent the strains computed with the active stress model with a dashed blue line and a triangular marker in Figure 9. The active stress formulation has a similar behavior in terms of circumferential strains where both orthotropic models fit the experimental data within one standard deviation. In the longitudinal and radial directions, the strains are more accurate using the active stress formulation of Haddad and Samani. ${ }^{17}$ However, the active stress formulation is unable of reproducing any of the shear strains. In particular, similarly to what obtained using the transversely isotropic active strain model, the circumferential-longitudinal strains $E_{c l}$ increase from the endocardium to the epicardium. This behavior is opposite to the one shown in the experimental data. The circumferential-radial shear strains are better captured but qualitatively far from the experimental data. However, a small in-plane rotation is evident in the correct direction. All the models herein considered, both active strain and active stress, are unsuited to predict the longitudinal-radial shear strains. The comparison of the strains is summarized in Table 3, where for each model and for each strain, the standard deviation of the error between the predicted strain and the mean experimental value is computed along the line considered in Figure 9, as follows:

$$
\operatorname{Error}\left(E_{j k}\right)=\frac{\sum_{i=1}^{N}\left(E_{j k}\left(x_{i}^{r e f}\right)-E_{j k}^{r e f}\left(x_{i}^{r e f}\right)\right)^{2}}{N} .
$$

Here, $x_{i}^{r e f}$ is the measurement point along the transmural line, the subscript indices $j$ and $k$ span the directions $\mathbf{c}, \mathbf{l}$, and $\mathbf{r}$, and $E_{j k}^{r e f}$ is the mean value of the experimental strain measurement (the solid black line in Figure 9). In the last column of Table 3, the error for the experimental data has been computed by substituting $E_{j k}\left(x_{i}^{r e f}\right)$ in (49) with the value of the black error bar at $x_{i}^{r e f}$ in Figure 9.

Figure 10 shows the stress state of the myocardium along the three orthotropic directions, $\mathbf{f}, \mathbf{s}$, and $\mathbf{n}$. Axial and shear stresses have been evaluated at the equatorial region of the ventricle at the end of systolic phase when $p_{v}$ reached $14 \mathrm{kPa}$. We notice in Figure 10A-C that the heterogeneous orthotropic activation model yields a more uniform distribution of axial stresses and therefore an overall lower stress state. This complies with the tendency of biological tissues to grow and remodel due to mechanical stimuli. A large variation in the stress distribution would lead to a growth in the regions subjected to greater loads and to a decrease of muscular tone in regions with smaller stresses. Since it is difficult, if not impossible, to measure the active stresses in the whole living myocardium, a homogeneity argument for the stresses can be used to interpret the results. Following this principle, the transversely isotropic activation model shows the largest transmural variation of the stresses. Normal stresses vary transmurally from -35 to $-15 \mathrm{kPa}$ along fibers, from -20 to $0 \mathrm{kPa}$ along sheet and from -25 to $0 \mathrm{kPa}$ along normal from the endocardium to the epicardium. $\sigma_{f s}$ and $\sigma_{s n}$ decrease from the endocardium to the epicardium from about 5 to $0 \mathrm{kPa}$, while $\sigma_{f n}$ is negative at the endocardium increases until reaching the peak of $7.5 \mathrm{kPa}$ around midwall and then decreases to $2 \mathrm{kPa}$ at the epicardium. The orthotropic activation model predict normal stresses ranging from -10 to $10 \mathrm{kPa}$ along the fibers, from -10 to $15 \mathrm{kPa}$ along the sheets, and from -15 to $0 \mathrm{kPa}$ along normal from the endocardium to the epicardium. $\sigma_{f s}$ varies from being slightly negative at the 


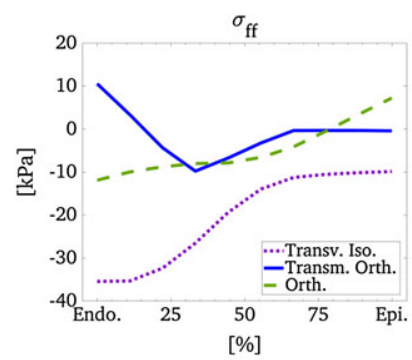

(A)

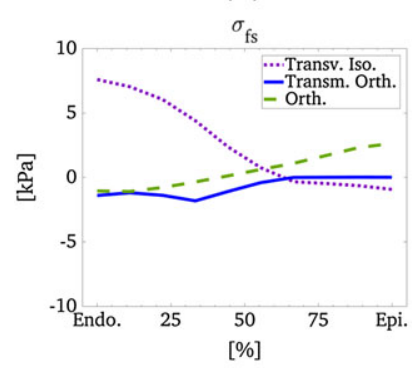

(D)

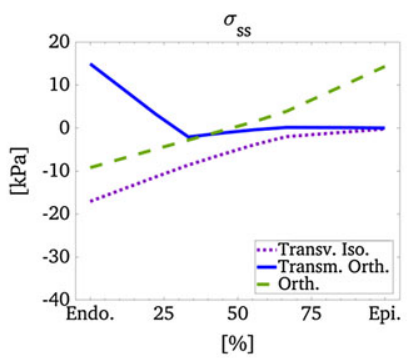

(B)

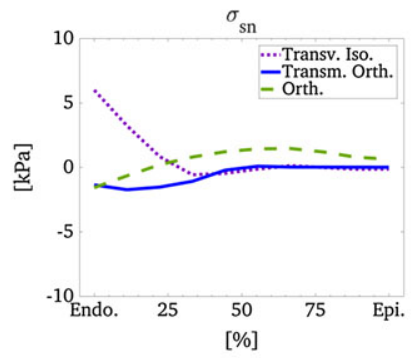

(E)

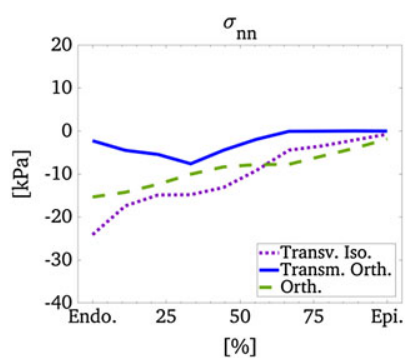

(C)

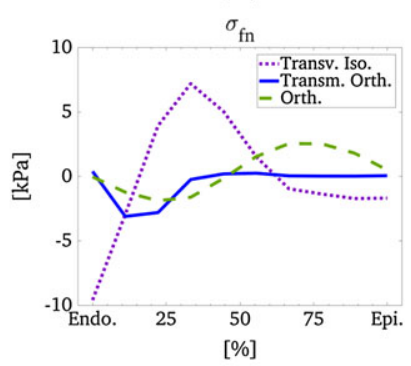

(F)

FIGURE 10 Systolic stresses at equatorial height with $p_{v}=14 \mathrm{kPa}$. The six independent components of the Cauchy stress tensor in material coordinates system $\left(\sigma_{f f}, \sigma_{s s}, \sigma_{n n}, \sigma_{f s}, \sigma_{s n}, \sigma_{f n}\right)$ are plotted over a normalized transmural coordinate. The three lines represent stresses for the transversely isotropic (..), orthotropic (-), and transmurally heterogeneous orthotropic (-) activation models. The top line displays the axial stresses along fibers, sheets, and normals. The bottom line displays the shear stresses

endocardium to $2.5 \mathrm{kPa}$ at epicardium, $\sigma_{s n}$ varies from $-2 \mathrm{kPa}$ at the endocardium to a barely positive value at epicardium, and $\sigma_{f n}$ is approximately null at both the endocardium and epicardium; it becomes negative in the subendocardial region, changes sign at midwall, and is positive in the subepicardial region. The transmurally heterogeneous orthotropic activation model presents normal stresses that are close to $0 \mathrm{kPa}$ at epicardium. $\sigma_{f f}$ decreases from $10 \mathrm{kPa}$ at the endocardium to the minimum of $-10 \mathrm{kPa}$ in the subendocardial region and then increases towards zero in the subendocardial region. $\sigma_{s s}$ decreases in the subendocardial region from $15 \mathrm{kPa}$ to a slightly negative value, which remains constant until epicardium. $\sigma_{n n}$ presents the most uniform distribution ranging from -2 to $0 \mathrm{kPa}$ from the endocardium to epicardium. Regarding shear stresses, $\sigma_{f s}$ and $\sigma_{s n}$ present a similar distribution, being slightly negative in the subendocardial region and approximately null in the subepicardial region. $\sigma_{f n}$ presents negative values in the subendocardial region with a peak of -3 and $0 \mathrm{kPa}$ at midwall and in the subepicardial region. The stress computed using the transmurally heterogeneous orthotropic activation presented in this work is generally more uniformly distributed.

\section{5 | Limitations of the proposed model}

Despite the several advantages of the proposed transmurally heterogenous orthotropic model, we discuss its (few) limitations.

We analyzed the mechanics of the left ventricle neglecting other coupled phenomena such as interaction with the fluid inside the cavity and the electrical activation of the myocardium. In particular, the latter assumption is motivated by the fact that, while there are regional differences in terms of electrical activation, the tension in the sarcomere increases slowly after electrical activation, determining a time delay between the electrical activation and the peak in contraction. This delay allows the left ventricle to contract almost uniformly. Therefore, when considering healthy conditions, a reasonable assumption, also adopted by several other authors (eg, Bovendeerd et $\mathrm{al}^{14}$ ), is to consider a uniform mechanical activation. However, as we have shown in Figure 6, this has a negligible effect on the results. Moreover, considering the apex-to-base and transmural delays without transmural variations in the mechanical activation is not sufficient to capture the qualitative behavior of the strains. For example, in Rossi et al, ${ }^{20}$ an electromechanical model of the heart using the orthotropic active strain model was used but showed opposite in-plane rotations and extremely large radial strains.

We considered an idealized geometry of the left ventricle, and we did not account for the presence of the right ventricle when setting the boundary conditions. Therefore, our predictions are axisymmetric and cannot represent the heterogeneity of a real geometry but must be thought as a strain prediction in the free wall area in agreement with the experimental measurements considered. 
We only considered the usual transmurally linear distribution of fiber ranging from $-60^{\circ}$ at epicardium to $60^{\circ}$ at the endocardium: Other choices might affect the considered strains.

Finally, the proposed transmurally heterogeneous orthotropic activation model relies on the assumption that there exists a rearranging mechanism of the fibers inside the collagen sheets and that this rearrangement is transmurally varying, although the results obtained using the model well represent the measured strains; the physiology of this assumption needs further studies in order to relate the transmural variation of the orthotropic parameter to physiological quantities.

\section{6 | CONCLUSIONS}

In this work, we proposed a new model for the active description of the ventricular contraction. To improve the agreement with experimental measurements of the transmural strains of current models of the active contraction of the left ventricle, we developed the novel transmurally heterogeneous orthotropic active strain model. In fact, the transversely isotropic active strain model, where the behavior of the isolated cell is modeled at the microscale, is unable to describe physiological wall thickening and ejection fraction at physiological level of fiber shortening. This fact led the author of the orthotropic active strain mode ${ }^{20}$ to note that myocytes embedded in the myocardium behaves differently from what observed in experiments on isolated myocytes. Consequently, they hypothesized that the myocardium behaves as a multiscale material due to the interplay of myocytes with the collagen network. This interplay has been thought as a rearranging mechanism of the myocytes within the collagen sheets: Simple geometrical assumptions link the fiber shortening with collagen inextensibility, thus resulting in an anisotropic multiscale description of the ventricular contraction. This multiscale description of the myocardium well captures the global wall thickening and the physiological ejection fraction. Nonetheless, the orthotropic active strain model cannot reproduce the transmural heterogeneities of systolic strains as observed in Rademakers et al. ${ }^{25}$ The transmural heterogeneities of the systolic strains have been revealed by experimental studies long ago $^{25}$ and suggested us to reformulate the rearranging mechanism of the original orthotropic active model introducing a transmurally varying level of anisotropy.

By numerically simulating the full cardiac cycle, we evaluated the wall strains and we compared the results with the experimental data in Omens et $\mathrm{al}^{51}$ and Waldman et al. ${ }^{54}$ We have shown through our numerical results the limitation of the orthotropic model ${ }^{20}$ in capturing the correct ventricular shortening and torsion. Similarly, we have shown the limitations of the transversely isotropic active strain model ${ }^{19}$ in capturing the circumferential strains and in-plane rotations. Instead, our proposed active strain model gives a better description of shear strains. In particular, it captures more accurately than the other models the strains related to rotation and torsion (circumferential-radial and circumferential-longitudinal), which are indicators of cardiac performance and have been proven to be sensitive to the presence of many pathologies, ${ }^{55-60}$ while maintaining the ability to describe large wall thickening. For reference, we have also reported the numerical results of a study that used an active stress model. ${ }^{17}$ The main limitation of the orthotropic active strain models is in the description of the longitudinal strain. In fact, the magnitude of this strain is larger than the measured one, although the transmurally heterogeneous orthotropic activation model improves the prediction of this strain. Indeed, the transmurally heterogeneous orthotropic activation model improves the prediction of circumferential strains and longitudinal strain of transversely isotropic and orthotropic activation models, respectively, while capturing correctly shear strains related to in-plane rotation and ventricular torsion. The behavior of the strains in the proposed transmurally heterogenous orthotropic model is in qualitative agreement with the experimental data.

We have also evaluated and compared the transmural stress distributions for all active strain models under consideration. Both orthotropic activation models provide axial stresses lower than the transversely isotropic activation model and a more uniform transmural distribution of the shear stresses. This is due to the description of the complex microstructure of the myocardium introduced by the orthotropic model, where the myocardial activation considers the interactions between myocytes and collagen sheets.

The effect of surrounding organs and tissues was modeled by means of a Robin boundary condition at epicardium. We performed a sensitivity analysis for the coefficient of the Robin boundary conditions used on the epicardial surface, and we confirmed that, as previously reported, ${ }^{52}$ the pericardium is important during diastole because it limits the ventricular compliance. We used such boundary condition also for the systolic phase. Although we did not consider the influence of different boundary conditions on the systolic strains, we believe that those may play a major role in determining the actual epicardial strains. In general, the type of boundary conditions to use in ventricular cardiac models still remains an open question. 
In conclusion, despite that the rearrangement mechanism of the fibers proposed is still to be proved experimentally, we have shown that the inclusion of a transmurally varying contraction mechanism plays a major role in determining systolic strains. The proposed transmurally heterogeneous orthotropic activation model describes more accurately than the other models ventricular contraction. Specifically, the proposed model provides more accurate strain distributions as shown by the validation against experimental measurements.

\section{ACKNOWLEDGMENTS}

The third and fourth authors acknowledge the ERC Advanced Grant iHEART, "An Integrated Heart Model for the simulation of the cardiac function", 2017-2022, P.I. A. Quarteroni (ERC-2016-ADG, project ID: 740132). The authors would like to thank Dr E. Faggiano and Prof D. Ambrosi for the precious support given at the beginning of this research. The authors would also like to thank Dr S. Pezzuto and Dr D. Bonomi for their help with the software and the mesh and the whole CMCS group at EPFL for all the stimulating discussions.

\section{ORCID}

\section{Luca Barbarotta (iD) http://orcid.org/0000-0003-3422-8014}

\section{REFERENCES}

1. Quarteroni A, Lassila T, Rossi S, Ruiz-Baier R. Integrated heart-coupling multiscale and multiphysics models for the simulation of the cardiac function. Comput Meth Appl Mech Eng. 2017;314:345-407.

2. Yancy CW, Jessup M, Bozkurt B, et al. 2013 ACCF/AHA guideline for the management of heart failure. Circulation. 2013;128(16):e240-e327.

3. Vigmond EJ, Clements C, McQueen DM, Peskin CS. Effect of bundle branch block on cardiac output: a whole heart simulation study. Prog Biophys Mol Biol. 2008;97(2):520-542.

4. Sugiura S, Washio T, Hatano A, Okada J, Watanabe H, Hisada T. Multi-scale simulations of cardiac electrophysiology and mechanics using the University of Tokyo heart simulator. Prog Biophys Mol Biol. 2012;110(2):380-389.

5. Baillargeon B, Rebelo N, Fox DD, Taylor RL, Kuhl E. The living heart project: a robust and integrative simulator for human heart function. Eur J Mech A Solids. 2014;48:38-47.

6. Crozier A, Augustin CM, Neic A, et al. Image-based personalization of cardiac anatomy for coupled electromechanical modeling. Ann Biomed Eng. 2016;44(1):58-70.

7. Lee J, Cookson A, Roy I, et al. Multiphysics computational modeling in CHeart. SIAM J Sci Comput. 2016;38(3):C150-C178.

8. McCulloch AD. Systems biophysics: multiscale biophysical modeling of organ systems. Biophys J. 2016;110(5):1023.

9. Quarteroni A, Manzoni A, Vergara C. The cardiovascular system: mathematical modelling, numerical algorithms and clinical applications. Acta Numer. 2017;26:365-590.

10. Nordsletten DA, Niederer SA, Nash MP, Hunter PJ, Smith NP. Coupling multi-physics models to cardiac mechanics. Prog Biophys Mol Biol. 2011;104(13):77-88.

11. Ponnaluri AVS, Perotti LE, Ennis DB, Klug WS. A viscoactive constitutive modeling framework with variational updates for the myocardium. Comput Meth Appl Mech Eng. 2017;314:85-101.

12. Eriksson TSE, Prassl AJ, Plank G, Holzapfel GA. Influence of myocardial fiber/sheet orientations on left ventricular mechanical contraction. Math Mech Solids. 2013;18(6):592-606.

13. Holzapfel GA, Ogden RW. Constitutive modelling of passive myocardium: a structurally based framework for material characterization. Philos Trans Royal Soc A: Math Phys Eng Sci. 2009;367(1902):3445-3475.

14. Bovendeerd PHM, Kroon W, Delhaas T. Determinants of left ventricular shear strain. Am J Physiol Heart Circ Physiol. 2009;297(3):H1058-68.

15. Bestel J, Clément F, Sorine M. A biomechanical model of muscle contraction. In: Niessen WJ, Viergever MA, eds. International Conference on Medical Image Computing and Computer-Assisted Intervention. Springer, Berlin, Heidelberg; 2001:1159-1161.

16. Guccione JM, Costa KD, McCulloch AD. Finite element stress analysis of left ventricular mechanics in the beating dog heart. $J$ Biomech. 1995;28(10):1167-1177.

17. Haddad SMH, Samani A. A computational model of the left ventricle biomechanics using a composite material approach. Int J Eng Sci. 2017;111:61-73.

18. Ambrosi D, Arioli G, Nobile F, Quarteroni A. Electromechanical coupling in cardiac dynamics: the active strain approach. SIAM J Appl Math. 2011;71(2):605-621.

19. Gjerald S, Hake J, Pezzuto S, Sundnes J, Wall ST. Patient-specific parameter estimation for a transversely isotropic active strain model of left ventricular mechanics. In: Camara O, Mansi T, Pop M, Rhode K, Sermesant M, Young A, eds. International Workshop on Statistical Atlases and Computational Models of the Heart. Berlin: Springer; 2014:93-104.

20. Rossi S, Lassila T, Ruiz-Baier R, Sequeira A, Quarteroni A. Thermodynamically consistent orthotropic activation model capturing ventricular systolic wall thickening in cardiac electromechanics. Eur J Mech A Solids. 2014;48:129-142. 
21. Gerbi A, Dedè L, Quarteroni A. A monolithic algorithm for the simulation of cardiac electromechanics in the human left ventricle. Math Eng. 2018;1(1):1-46.

22. Göktepe S, Menzel A, Kuhl E. The generalized Hill model: a kinematic approach towards active muscle contraction. J Mech Phys Solids. 2014;72:20-39.

23. Stålhand J, Klarbring A, Holzapfel GA. A mechanochemical 3D continuum model for smooth muscle contraction under finite strains. $J$ Theor Biol. 2011;268(1):120-130.

24. Pezzuto S. Mechanics of the heart: constitutive issues and numerical experiments. Ph.D. Thesis: Politecnico di Milano; 2013.

25. Rademakers FE, Rogers WJ, Guier WH, et al. Relation of regional cross-fiber shortening to wall thickening in the intact heart. Three-dimensional strain analysis by NMR tagging. Circulation. 1994;89(3):1174-1182.

26. Rossi S, Ruiz-Baier R, Pavarino LF, Quarteroni A. Orthotropic active strain models for the numerical simulation of cardiac biomechanics. Int J Numer Method Biomed Eng. 2012;28(6-7):761-788.

27. Ciarlet PG. The Finite Element Method for Elliptic Problems. Philadelphia, Pa: SIAM; 2002.

28. Simo JC, Taylor RL. Penalty function formulations for incompressible nonlinear elastostatics. Comput Meth Appl Mech Eng. 1982;35(1):107-118.

29. Schröder J, Viebahn N, Balzani D, Wriggers P. A novel mixed finite element for finite anisotropic elasticity; the SKA-element simplified kinematics for anisotropy. Comput Meth Appl Mech Eng. 2016;310:475-494.

30. Nash MP, Hunter PJ. Computational mechanics of the heart. J Elast Phys Sci Solids. 2000;61(1-3):113-141.

31. Nash MP, Panfilov AV. Electromechanical model of excitable tissue to study reentrant cardiac arrhythmias. Prog Biophys Mol Biol. 2004;85(2-3):501-522.

32. Costa KD, Holmes JW, Mcculloch AD. Modelling cardiac mechanical properties in three dimensions. Philos Trans R Soc London A: Math Phys Eng Sci. 2001;359(1783):1233-1250.

33. Fung YC. Mathematical representation of the mechanical properties of the heart muscle. J Biomech. 1970;3(4):381-404

34. Legrice IJ, Hunter PJ, Smaill BH. Laminar structure of the heart: a mathematical model. Am J Physiol Heart Circ Physiol. 1997;272(5):H2466-H2476.

35. Holzapfel GA. Nonlinear Solid Mechanics. Chichester: Wiley; 2000.

36. Garrison P. Basic Structures. Chichester: John Wiley \& Sons; 2016.

37. Flory PJ. Thermodynamic relations for high elastic materials. Trans Faraday Soc. 1961;57:829-838.

38. Sansour C. On the physical assumptions underlying the volumetric-isochoric split and the case of anisotropy. Eur J Mech A Solids. 2008;27(1):28-39.

39. Helfenstein J, Jabareen M, Mazza E, Govindjee S. On non-physical response in models for fiber-reinforced hyperelastic materials. Int J Solids Struct. 2010;47(16):2056-2061.

40. Hartmann S, Neff P. Polyconvexity of generalized polynomial-type hyperelastic strain energy functions for near-incompressibility. Int $J$ Solids Struct. 2003;40(11):2767-2791.

41. Simo JC, Taylor RL. Quasi-incompressible finite elasticity in principal stretches. Continuum basis and numerical algorithms. Comput Meth Appl Mech Eng. 1991;85(3):273-310.

42. Boyett MR, Frampton JE, Kirby MS. The length, width and volume of isolated rat and ferret ventricular myocytes during twitch contractions and changes in osmotic strength. Exp Physiol. 1991;76(2):259-270.

43. Quinn TA, Kohl P. Combining wet and dry research: experience with model development for cardiac mechano-electric structure-function studies. Cardiovasc Res. 2013;97(4):601-611.

44. Wong J, Kuhl E. Generating fibre orientation maps in human heart models using Poisson interpolation. Comput Meth Biomech Biomed Eng. 2014;17(11):1217-1226.

45. Geuzaine C, Remacle JF. Gmsh: A 3-D finite element mesh generator with built-in pre-and post-processing facilities. Int J Numer Methods Eng. 2009;79(11):1309-1331.

46. Ben-Israel A. A Newton-Raphson method for the solution of systems of equations. J Math Anal Appl. 1966;15(2):243-252.

47. Quarteroni A, Sacco R, Saleri F. Numerical Mathematics. Berlin Heidelberg: Springer Science \& Business Media; 2010.

48. Quarteroni A, Valli A. Domain Decomposition Methods for Partial Differential Equations. Oxford, UK: Oxford University Press; 1999.

49. Tricerri P, Dedè L, Gambaruto A, Quarteroni A, Sequeira A. A numerical study of isotropic and anisotropic constitutive models with relevance to healthy and unhealthy cerebral arterial tissues. Int J Eng Sci. 2016;101:126-155.

50. Westerhof N, Lankhaar JW, Westerhof BE. The arterial windkessel. Med Biol Eng Comput. 2009Feb;47(2):131-141.

51. Omens JH, May KD, McCulloch AD. Transmural distribution of three-dimensional strain in the isolated arrested canine left ventricle. Am J Physiol Heart Circ Physiol. 1991;261(3):H918-H928.

52. Glantz SA, Misbach GA, Moores WY, et al. The pericardium substantially affects the left ventricular diastolic pressure-volume relationship in the dog. Circ Res. 1978;42(3):433-441.

53. Ramanathan C, Jia P, Ghanem R, Ryu K, Rudy Y. Activation and repolarization of the normal human heart under complete physiological conditions. Proc Nat Acad Sci. 2006;103(16):6309-6314.

54. Waldman LK, Nosan D, Villarreal F, Covell JW. Relation between transmural deformation and local myofiber direction in canine left ventricle. Circ Res. 1988;63(3):550-562.

55. Young AA, Cowan BR. Evaluation of left ventricular torsion by cardiovascular magnetic resonance. J Cardiovasc Magn Reson. 2012;14(1):49. 
56. Kanzaki H, Nakatani S, Yamada N, Urayama S, Miyatake K, Kitakaze M. Impaired systolic torsion in dilated cardiomyopathy: reversal of apical rotation at mid-systole characterized with magnetic resonance tagging method. Basic Res Cardiol. 2006;101(6):465-470.

57. Fonseca CG, Dissanayake AM, Doughty RN, et al. Three-dimensional assessment of left ventricular systolic strain in patients with type 2 diabetes mellitus, diastolic dysfunction, and normal ejection fraction. Am J Cardiol. 2004;94(11):1391-1395.

58. Nagel E, Stuber M, Burkhard B, et al. Cardiac rotation and relaxation in patients with aortic valve stenosis. Eur Heart J. 2000;21(7):582-589.

59. Bertini M, Delgado V, Nucifora G, et al. Left ventricular rotational mechanics in patients with coronary artery disease: differences in subendocardial and subepicardial layers. Heart. 2010;96(21):1737-1743.

60. Lumens J, Delhaas T, Arts T, Cowan BR, Young AA. Impaired subendocardial contractile myofiber function in asymptomatic aged humans, as detected using mri. Am J Physiol Heart Circ Physiol. 2006;291(4):H1573-H1579.

How to cite this article: Barbarotta L, Rossi S, Dedè L, Quarteroni A. A transmurally heterogeneous orthotropic activation model for ventricular contraction and its numerical validation. Int J Numer Meth Biomed Engng. 2018;34:e3137. https://doi.org/10.1002/cnm.3137 